TRANSACTIONS OF THE

AMERICAN MATHEMATICAL SOCIETY

Volume 354, Number 2, Pages 749-776

S 0002-9947(01)02916-6

Article electronically published on October 3, 2001

\title{
NEW BASES FOR TRIEBEL-LIZORKIN AND BESOV SPACES
}

\author{
G. KYRIAZIS AND P. PETRUSHEV
}

\begin{abstract}
We give a new method for construction of unconditional bases for general classes of Triebel-Lizorkin and Besov spaces. These include the $L_{p}$, $H_{p}$, potential, and Sobolev spaces. The main feature of our method is that the character of the basis functions can be prescribed in a very general way. In particular, if $\Phi$ is any sufficiently smooth and rapidly decaying function, then our method constructs a basis whose elements are linear combinations of a fixed (small) number of shifts and dilates of the single function $\Phi$. Typical examples of such $\Phi$ 's are the rational function $\Phi(\cdot)=\left(1+|\cdot|^{2}\right)^{-N}$ and the Gaussian function $\Phi(\cdot)=e^{-|\cdot|^{2}}$. This paper also shows how the new bases can be utilized in nonlinear approximation.
\end{abstract}

\section{INTRODUCTION}

The construction of unconditional bases for a given function space is important in many applications. Such bases frequently lead to a simple characterization of this space in terms of norms applied to the sequence of coefficients with respect to that basis. The sequence norm characterization then permits the solution of extremal problems. Unconditional bases in this context have been used in many fields such as statistics [Do], image processing [DJKP, nonlinear approximation De, and functional analysis (for example the characterization of K-functionals).

From many perspectives, it is of great benefit if one can prescribe the nature of these bases. For example, wavelet bases are popular because of their timefrequency localization. We are interested therefore in constructing bases in which the character of the basis functions is prescribed in advance. In this paper we put forward a method which allows this flexibility. Our main application in this paper is to the construction of bases generated from a small number of shifts and dilates of a single function $\Phi$.

Our method was first introduced in $[\overline{\mathrm{Pet}}]$ in the restricted setting of univariate functions in $L_{p}$ and $C$. In the present paper, we will extend this construction to multivariate functions. We will also establish our results under minimal and natural assumptions. Moreover, we will establish that these bases are unconditional for a wide range of function spaces, namely any spaces from the classes of Triebel-Lizorkin or Besov spaces.

We will use the remainder of this introduction to give our construction of bases and for a more detailed outline of the contents of this paper.

Received by the editors June 24, 1999.

1991 Mathematics Subject Classification. Primary 41A17, 41A20, 42B25, 42C15.

Key words and phrases. Triebel-Lizorkin spaces, Besov spaces, unconditional bases, nonlinear approximation, wavelets.

This research was supported by ARO Research Contract DAAG55-98-1-0002.

(C)2001 American Mathematical Society 
Throughout this paper, we use standard multi-index notation. In particular, for every $x=\left(x_{1}, \ldots, x_{d}\right) \in \mathbb{R}^{d}$ and $\alpha=\left(\alpha_{1}, \ldots, \alpha_{d}\right) \in \mathbb{Z}_{+}^{d}\left(\mathbb{Z}_{+}:=\{n: n \geq 0\}, d \geq 1\right)$, we let $|x|:=\sqrt{x_{1}^{2}+\cdots+x_{d}^{2}}, x^{\alpha}:=x_{1}^{\alpha_{1}} \cdots x_{d}^{\alpha_{d}},|\alpha|:=\alpha_{1}+\cdots+\alpha_{d}, \alpha !=\alpha_{1} ! \cdots \alpha_{d} !$, and $(\cdot)^{\alpha}:=\frac{\partial^{|\alpha|}(\cdot)}{\partial^{\alpha} x_{1} \cdots \partial^{\alpha} d x_{d}}$.

We denote by $\mathcal{S}:=\mathcal{S}\left(\mathbb{R}^{d}\right)$ the Schwartz space of infinitely differentiable, rapidly decreasing functions on $\mathbb{R}^{d}$ and by $\mathcal{S}^{\prime}:=\mathcal{S}^{\prime}\left(\mathbb{R}^{d}\right)$ its dual, the space of tempered distributions. We also denote by $\mathcal{S}^{\prime} / \mathcal{P}$ the space of equivalence classes of distributions in $\mathcal{S}^{\prime}$ modulo polynomials, i.e., $\mathcal{S}^{\prime} / \mathcal{P}$ is the dual of the space $\mathcal{S}_{\infty}:=\mathcal{S}_{\infty}\left(\mathbb{R}^{d}\right)$ of all functions $\eta \in \mathcal{S}$ such that $\int \eta(x) x^{\alpha} d x=0$ for $\alpha \in \mathbb{Z}_{+}^{d}$.

We write $D$ for the family of all dyadic cubes in $\mathbb{R}^{d}$ and $D_{m}, m \in \mathbb{Z}$, for the collection of all cubes $I \in D$ of side-length $\ell(I)=2^{-m}$. For any dyadic cube $I \in D$, we use $x_{I}$ for its lower-left corner and $|I|$ for its volume. Finally, for any distribution $f \in \mathcal{S}^{\prime}$, we define

$$
f_{I}(\cdot):=|I|^{-1 / 2} f\left(\frac{\cdot-x_{I}}{\ell(I)}\right)
$$

where the dilation and translation are considered in distributional sense.

1.1. Wavelets. Wavelets will be used as a tool for our construction of new bases. We recall, without elaboration, some of the fundamental results on wavelets. A more detailed discussion can be found in Meyer [M] or Daubechies [Da].

Multivariate wavelet bases are typically constructed as tensor products of a univariate scaling function $\psi^{0}:=\phi$ and associated wavelet $\psi$. Namely, let $E$ $\left(\# E=2^{d}-1\right)$ denote the set of nonzero vertices of the unit cube in $\mathbb{R}^{d}$. For each vertex $e=\left(e_{1}, \cdots, e_{d}\right) \in E$ we let

$$
\psi^{e}(x):=\psi^{e_{1}}\left(x_{1}\right) \ldots \psi^{e_{d}}\left(x_{d}\right)
$$

and define $\Psi:=\left\{\psi^{e}: e \in E\right\}$. Then the collection

$$
W:=\left\{\psi_{I}^{e}: I \in D, e \in E\right\}
$$

forms an orthonormal basis for the space $L_{2}\left(\mathbb{R}^{d}\right)$.

Another way of constructing tensor product wavelet bases is by starting at a certain dyadic level, for instance $D_{0}$, and using again the dilates and shifts of $\Psi$. Namely, let

$$
\Psi_{0}:=\Psi \cup\left\{\psi^{0}\right\}=\left\{\psi^{e}: e \in E_{0}\right\}
$$

where $E_{0}:=E \cup\{0\}$ with 0 the zero vector in $\mathbb{R}^{d}$. Now

$$
W_{0}:=\left\{\psi_{I}^{0}: I \in D_{0}\right\} \cup\left\{\psi_{I}^{e}: I \in D^{+}, e \in E\right\} \quad \text { with } \quad D^{+}:=\bigcup_{m \geq 0} D_{m}
$$

is an orthonormal basis for $L_{2}\left(\mathbb{R}^{d}\right)$.

Standard assumptions on the set $\Psi\left(\Psi_{0}\right)$ include

A1. $\Psi \subset C^{r}\left(\mathbb{R}^{d}\right)$ and

$$
\left|\left(\psi^{e}\right)^{(\alpha)}(x)\right| \leq C(1+|x|)^{-M}, \quad|\alpha| \leq r, \quad e \in E\left(e \in E_{0}\right) .
$$

A2.

$$
\int_{\mathbb{R}^{d}} x^{\alpha} \psi^{e}(x) d x=0, \quad|\alpha|<r, \quad e \in E
$$

For instance, starting with Meyer's univariate scaling function and wavelet one obtains a basis of functions from $\mathcal{S}_{\infty}$ which satisfy A1-2 for any selection of the parameters $r$ and $M$. Another example of a wavelet set satisfying A1-2 is provided 
by starting with any univariate compactly supported wavelet and scaling function which are smooth enough. In what follows, we will always use the tensor products of Meyer's wavelets unless otherwise mentioned.

By varying the smoothness and decay parameters $r$ and $M$, one can prove that $W$ forms an unconditional basis for a host of distribution spaces such as $L_{p}:=$ $L_{p}\left(\mathbb{R}^{d}\right)(1<p<\infty), H_{p}:=H_{p}\left(\mathbb{R}^{d}\right)(0<p \leq 1)$, or the more general homogeneous Triebel-Lizorkin and Besov spaces $\dot{F}_{p q}^{s}$ and $\dot{B}_{p q}^{s}$ (see the Appendix).

In particular, if $r$ and $M$ are sufficiently large (depending on the parameters $s \in \mathbb{R}, 0<p<\infty$, and $0<q \leq \infty)$, then for every $f \in \dot{F}_{p q}^{s}$ there exist unique coefficients $c_{I e}(f),(I, e) \in D \times E$, such that

$$
f=\sum_{I \in D} \sum_{e \in E} c_{I e}(f) \psi_{I}^{e} \text { with } c_{I e}(f):=\left\langle f, \psi_{I}^{e}\right\rangle,
$$

where the convergence is considered in the sense of $\mathcal{S}^{\prime} / \mathcal{P}$ (and in the sense of $\dot{F}_{p q}^{s}$ when $q \neq \infty$ ). Moreover, the following wavelet characterization holds

$$
\|f\|_{\dot{F}_{p q}^{s}} \approx\left\|\left(\sum_{I, e}\left(|I|^{-s / d}\left|c_{I e}(f) \lambda_{I}\right|\right)^{q}\right)^{1 / q}\right\|_{L_{p}}
$$

with the usual modification when $q=\infty$, where $\lambda_{I}:=|I|^{-1 / 2} \chi_{I}$ is the characteristic function of $I$ normalized in $L_{2}$. Here we have adopted the notation $A \approx B$ which means that there exist constants $C_{1}, C_{2}>0$ such that $C_{1} A \leq B \leq C_{2} A$. The equivalence constants $C_{1}$ and $C_{2}$ in (1.2) depend on $d, p, q$, and $s$. On other occasions, the reader will have to consult the text to understand the parameters on which the equivalence constants depend on. Throughout the paper, the constants are denoted by $C, C_{1}, \ldots$ and they may vary at every occurrence.

Similarly, (for suitable $r$ and $M$ ) for every $f \in \dot{B}_{p q}^{s}, s \in \mathbb{R}, 0<p, q \leq \infty$, the representation (1.1) holds with convergence considered in the sense of $\mathcal{S}^{\prime} / \mathcal{P}$ (and in the sense of $\dot{B}_{p q}^{s}$ when $\left.p, q \neq \infty\right)$. Also

$$
\|f\|_{\dot{B}_{p q}^{s}} \approx\left(\sum_{e \in E} \sum_{m \in \mathbb{Z}}\left(\sum_{I \in D_{m}}\left(|I|^{-s / d+1 / p-1 / 2}\left|c_{I e}(f)\right|\right)^{p}\right)^{q / p}\right)^{1 / q}
$$

with the usual modifications when $q=\infty$ or $p=\infty$.

The characterization of Besov spaces (1.3) has been proved by several authors in various subcases (see [M], [K1], and the references therein) under different assumptions. For the Triebel-Lizorkin spaces, we refer the reader to [FJW] and [K3]. For more details, see the Appendix. We would like also to point out that technically speaking, our systems will be the bases for the Triebel-Lizorkin and Besov spaces provided that $p, q \neq \infty$, since in that case the convergence of the corresponding basic series will take place in the quasi-norm of the space in question. However, with a slight abuse of the terminology we use the word bases even if $p$ or $q=\infty$, where the convergence is considered only in the distributional sense.

1.2. The construction of new bases for homogeneous spaces. Wavelets are functions of a rather particular nature. It is the goal of this paper to give a new scheme for the construction of basis consisting of functions of a more general nature. The idea of our construction stems from the well known principle of a small perturbation argument. That is, to approximate the elements of a given good basis by the elements of the new basis. The fundamental question is: What kind of 
approximation should be used for this construction? In what follows, we present our approach to this question.

Let $\varepsilon>0$ and let $\Theta:=\left\{\theta^{e}: e \in E\right\}$ be a subset of $C^{r}\left(\mathbb{R}^{d}\right)$ satisfying the following conditions:

$$
\begin{gathered}
\left|\left(\psi^{e}\right)^{(\alpha)}(x)-\left(\theta^{e}\right)^{(\alpha)}(x)\right| \leq \varepsilon(1+|x|)^{-M}, \quad|\alpha| \leq r, \quad e \in E, \\
\int_{\mathbb{R}^{d}} x^{\alpha} \theta^{e}(x) d x=0, \quad|\alpha|<r, \quad e \in E
\end{gathered}
$$

where $\Psi:=\left\{\psi^{e}: e \in E\right\}$ is a wavelet set satisfying A1-2. We will show that the collection of functions

$$
B:=\left\{\theta_{I}^{e}: I \in D, e \in E\right\}
$$

is an unconditional bases for various function spaces. We call $B$ the new system (basis).

By scaling, using A1-2, (1.4), and (1.5), we obtain, for $I \in D, e \in E$,

B1.

$$
\left|\left(\psi_{I}^{e}\right)^{(\alpha)}(x)-\left(\theta_{I}^{e}\right)^{(\alpha)}(x)\right| \leq \varepsilon|I|^{-1 / 2-|\alpha| / d}\left(1+\frac{\left|x-x_{I}\right|}{\ell(I)}\right)^{-M}, \quad|\alpha| \leq r .
$$

B2.

$$
\left|\left(\theta_{I}^{e}\right)^{(\alpha)}(x)\right|,\left|\left(\psi_{I}^{e}\right)^{(\alpha)}(x)\right| \leq C|I|^{-1 / 2-|\alpha| / d}\left(1+\frac{\left|x-x_{I}\right|}{\ell(I)}\right)^{-M}, \quad|\alpha| \leq r .
$$

B3.

$$
\int_{\mathbb{R}^{d}} x^{\alpha} \theta_{I}^{e}(x) d x=\int_{\mathbb{R}^{d}} x^{\alpha} \psi_{I}^{e}(x) d x=0, \quad|\alpha|<r .
$$

These are the "small perturbation" properties of the new system that will enable us to prove that, for the full range of the indexes $s, p$, and $q$, for sufficiently small $\varepsilon$ the new system $B$ is an unconditional basis for $\dot{F}_{p q}^{s}$ and $\dot{B}_{p q}^{s}$ (see Theorems 3.1 3.2 below). That is, for every $f \in \dot{F}_{p q}^{s}$ or $\dot{B}_{p q}^{s}$ there exist unique coefficients $d_{I, e}, I \in D, e \in E$, such that $f=\sum_{I, e} d_{I e} \theta_{I}^{e}$ and

$$
\|f\|_{\dot{F}_{p q}^{s}} \approx\left\|\left(\sum_{I, e}\left(|I|^{-s / d}\left|d_{I e} \lambda_{I}\right|\right)^{q}\right)^{1 / q}\right\|_{L_{p}}
$$

or

$$
\|f\|_{\dot{B}_{p q}^{s}} \approx\left(\sum_{e \in E} \sum_{m \in \mathbb{Z}}\left(\sum_{I \in D_{m}}\left(|I|^{-s / d+1 / p-1 / 2}\left|d_{I e}\right|\right)^{p}\right)^{q / p}\right)^{1 / q}
$$

respectively.

We recall that, for $1<p<\infty, \dot{F}_{p 2}^{0} \approx L_{p}$ while, for $0<p \leq 1, \dot{F}_{p 2}^{0} \approx H_{p}$ the real Hardy spaces, where $\approx$ means equivalent (with equivalent norms). Also, for $s>0,1<p<\infty, \dot{F}_{p 2}^{s} \approx H_{p}^{s}$ the potential space, and for integer values of $s, \dot{F}_{p 2}^{s}$ is equivalent to the usual Sobolev space $W_{p}^{s}$ equipped with its seminorm (see [T]). As a consequence, our construction gives unconditional bases for the above-mentioned spaces. 
1.3. The construction of new bases for inhomogeneous spaces. We now use a wavelet set $\Psi_{0}=\left\{\psi^{e}: e \in E_{0}\right\}$ satisfying A1-2. Let $\varepsilon>0$ and let $\Theta_{0}:=$ $\left\{\theta^{e}: e \in E_{0}\right\}$ be a subset of $C^{r}\left(\mathbb{R}^{d}\right)$ satisfying (1.4) and (1.5), and in addition to this

$$
\left|\left(\psi^{0}\right)^{(\alpha)}(x)-\left(\theta^{0}\right)^{(\alpha)}(x)\right| \leq \varepsilon(1+|x|)^{-M}, \quad|\alpha| \leq r .
$$

We define the new system by

$$
B_{0}:=\left\{\theta_{I}^{0}: I \in D_{0}\right\} \cup\left\{\theta_{I}^{e}: I \in D^{+}, e \in E\right\} .
$$

In this paper, we treat in detail only the homogeneous spaces. Similarly as in the homogeneous case it can be proved that for a suitable choice of the parameters $r$ and $M$, and the wavelet set $\Psi_{0}$ the new basis $B_{0}$ is an unconditional basis for the inhomogeneous Triebel-Lizorkin and Besov spaces $F_{p q}^{s}$ and $B_{p q}^{s}$. These are essentially the same results. The difference is that instead of using all dyadic levels $D$ we use the levels $D^{+}$. We leave the details of the inhomogeneous case to the reader.

As we have already mentioned our basic application is to the construction of bases from shifts and dilates of a single function $\Phi$. Armed with Theorems [3.1] 3.2 it remains only to understand for which functions $\Phi$ we have properties (1.4.1.5) for some finite linear combinations $\theta^{e}, e \in E\left(E_{0}\right)$ of shifts and dilates of $\Phi$. We prove two results which give sufficient conditions on $\Phi$ so that this is true. In Theorem 4.2 we show that any sufficiently smooth and rapidly decaying function $\Phi$ can do the job. The rational function $\Phi(\cdot)=\left(1+|\cdot|^{2}\right)^{-N}$ and the exponential function $\Phi(\cdot)=e^{-|\cdot|^{2}}$ are interesting examples of functions that satisfy these conditions. Consequently, we obtain rational and exponential bases. In Theorem 4.4 we give a second (very simple) construction of new bases in the specific case when the linear span of the shifts $\Phi(\cdot-j)$ contains the polynomials of a fixed degree. This type of functions plays an important role in the study of the approximation properties of shift-invariant spaces generated by piecewise polynomial functions or radial basis functions (see $[\mathrm{BR}]$ ). Moreover, the original construction of wavelets is based upon a function $\Phi$ whose shifts reproduce polynomials. However, additional assumptions on $\Phi$ are required such as the stability of the shifts of $\Phi$ and a refinement equation $\Phi(\cdot)=\sum_{j \in \mathbb{Z}^{d}} a_{j} \Phi(2 \cdot-j)$, conditions that we will not need to assume.

1.4. Nonlinear approximation. Unconditional bases for $L_{p}, H_{p}$, Besov and other spaces are of significant importance for nonlinear approximation. They provide a simple and powerful tool for approximation. We utilize our result to the theory of $n$-term nonlinear approximation from shifts and dilates of a single function $\Phi$ and, in particular, to multivariate rational approximation (see Theorem 5.2 and Corollary 5.1).

1.5. Outline of the paper. The outline of the paper is as follows: In $\S 2$, we give the necessary auxiliary results which we use to prove our basic results in $\S 3$. In $\S 4$, we apply our main results to the construction of bases from shifts and dilates of a single function. In $\S 5$, we utilize the new bases to $n$-term nonlinear approximation. $\S 6$ is an appendix, where we give the characterization of the homogeneous TriebelLizorkin and Besov spaces spaces $\dot{F}_{p q}^{s}$ and $\dot{B}_{p q}^{s}$ by wavelets. 


\section{SOME AUXILIARY RESUlts}

To prove that the new system $B$ is a basis for the spaces $\dot{F}_{p q}^{s}$ and $\dot{B}_{p q}^{s}$, we will use that Meyer's wavelets (or other suitable wavelets) constitute a basis for these spaces (see Appendix). Namely, we will use that the norms in $\dot{F}_{p q}^{s}$ and $\dot{B}_{p q}^{s}$ can be characterized by the discrete $\dot{f}_{p q}^{s}$ and $\dot{b}_{p q}^{s}$ norms of the wavelet coefficients of the functions, see (1.2) and (1.3).

We now recall the definition of the sequence spaces $\dot{f}_{p q}^{s}$ and $\dot{b}_{p q}^{s}$ (see [FJW]):

(a) For $s \in \mathbb{R}, 0<p<\infty$, and $0<q \leq \infty$, $\dot{f}_{p q}^{s}$ is defined as the family of all sequences $h:=\left(h_{I e}\right)_{(I, e) \in D \times E}$ such that

$$
\|h\|_{\dot{f}_{p q}^{s}}:=\left\|\left(\sum_{I, e}\left(|I|^{-s / d}\left|h_{I e} \lambda_{I}\right|\right)^{q}\right)^{1 / q}\right\|_{L_{p}}<\infty
$$

(b) For $s \in \mathbb{R}$, and $0<p, q \leq \infty, \dot{b}_{p q}^{s}$ is defined as the space of all sequences $h:=\left(h_{I e}\right)_{(I, e) \in D \times E}$ such that

$$
\|h\|_{\dot{b}_{p q}^{s}}:=\left(\sum_{e \in E} \sum_{m \in \mathbb{Z}}\left(\sum_{I \in D_{m}}\left(|I|^{-s / d+1 / p-1 / 2}\left|h_{I e}\right|\right)^{p}\right)^{q / p}\right)^{1 / q}<\infty .
$$

Since the wavelet family $W=\left\{\psi_{I}^{e}: I \in D, e \in E\right\}$ forms an orthonormal basis for $L_{2}\left(\mathbb{R}^{d}\right)$, we have, for every $(I, e) \in D \times E$,

$$
\theta_{I}^{e}=\sum_{J, e^{\prime}} a\left(I e, J e^{\prime}\right) \psi_{J}^{e^{\prime}}, \quad a\left(I e, J e^{\prime}\right):=\left\langle\theta_{I}^{e}, \psi_{J}^{e^{\prime}}\right\rangle .
$$

We will next show that the transformation matrix

$$
\mathbf{A}:=\mathbf{A}_{\varepsilon}:=\left(a\left(I e, J e^{\prime}\right)\right)_{(I, e),\left(J, e^{\prime}\right) \in D \times E}
$$

which maps the wavelet basis $W$ onto the new basis $B$ is very close to the identity matrix and this will give us everything we need.

Theorem 2.1. (a) Let $s \in \mathbb{R}, 0<p<\infty, 0<q \leq \infty, \mathcal{J}:=d / \min \{1, p, q\}$, $r>\max \{\mathcal{J}-d-s, s\}$, and $M>\max \{\mathcal{J}, d+r\}$. Then for sufficiently small $\varepsilon>0\left(0<\varepsilon \leq \varepsilon_{0}\right)$ the matrix $\mathbf{A}_{\varepsilon}$ is invertible and the operators associated with $\mathbf{A}_{\varepsilon}$ and $\mathbf{A}_{\varepsilon}^{-1}$ are bounded on $\dot{f}_{p q}^{s}$. The same holds for $\mathbf{A}_{\varepsilon}^{T}$ (the transpose of $\mathbf{A}_{\varepsilon}$ ) and $\left(\mathbf{A}_{\varepsilon}^{T}\right)^{-1}=\left(\mathbf{A}_{\varepsilon}^{-1}\right)^{T}$, respectively.

(b) Let $s \in \mathbb{R}, 0<p, q \leq \infty, \mathcal{J}:=d / \min \{1, p\}, r>\max \{\mathcal{J}-d-s, s\}$, and $M>\max \{\mathcal{J}, d+r\}$. Then for sufficiently small $\varepsilon>0$ the matrix $\mathbf{A}_{\varepsilon}$ is invertible, and $\mathbf{A}_{\varepsilon}$ and $\mathbf{A}_{\varepsilon}^{-1}$ are bounded on $\dot{b}_{p q}^{s}$. The same is true for $\mathbf{A}_{\varepsilon}^{T}$ and $\left(\mathbf{A}_{\varepsilon}^{T}\right)^{-1}$.

To avoid some long and tedious calculations, we will use for the proof of Theorem 2.1 the machinery of the almost diagonal matrices, developed in [FJ2] and [FJW].

Definition 2.1. The infinite matrix

$$
\mathbf{A}:=\left(a\left(I e, J e^{\prime}\right)\right)_{(I, e),\left(J, e^{\prime}\right) \in D \times E}
$$

is said to be almost diagonal on $\dot{f}_{p q}^{s}$ or $\dot{b}_{p q}^{s}$ if there exist $\delta>0$ and $C>0$ such that

$$
\left|a\left(I e, J e^{\prime}\right)\right| \leq C \omega_{\delta}(I, J), \quad(I, e),\left(J, e^{\prime}\right) \in D \times E,
$$


with

$$
\begin{aligned}
\omega_{\delta}(I, J) & :=\left(\frac{\ell(I)}{\ell(J)}\right)^{s}\left(1+\frac{\left|x_{I}-x_{J}\right|}{\max \{\ell(I), \ell(J)\}}\right)^{-\mathcal{J}-\delta} \\
& \times \min \left\{\left(\frac{\ell(I)}{\ell(J)}\right)^{(d+\delta) / 2},\left(\frac{\ell(J)}{\ell(I)}\right)^{(\delta-d) / 2+\mathcal{J}}\right\},
\end{aligned}
$$

where $\mathcal{J}:=d / \min \{1, p, q\}$ for $\dot{f}_{p q}^{s}$ and $\mathcal{J}:=d / \min \{1, p\}$ for $\dot{b}_{p q}^{s}$.

We will denote by $\mathbf{a d}_{p q}^{s}$ the family of all almost diagonal matrices on $\dot{f}_{p q}^{s}$ or $\dot{b}_{p q}^{s}$.

Proposition 2.1. Let $s \in \mathbb{R}, 0<p, q \leq \infty$, and $\mathbf{A} \in \mathbf{a d}_{p q}^{s}$. Then $\mathbf{A}$ is bounded on $\dot{b}_{p q}^{s}$ and, if $p<\infty$, on $\dot{f}_{p q}^{s}$.

Given an almost diagonal matrix

$$
\mathbf{A}:=\left(a\left(I e, J e^{\prime}\right)\right)_{(I, e),\left(J, e^{\prime}\right) \in D \times E},
$$

we define

$$
\|\mathbf{A}\|_{\delta}:=\sup _{I e, J e^{\prime}}\left|a\left(I e, J e^{\prime}\right)\right| / \omega_{\delta}(I, J) .
$$

Proposition 2.2. For any $\delta>0$ there exists $\varepsilon>0$ such that if $\mathbf{A} \in \mathbf{a d}_{p q}^{s}$ (on $\dot{f}_{p q}^{s}$ or $\left.\dot{b}_{p q}^{s}\right)$ and $\|\mathbf{I}-\mathbf{A}\|_{\delta}<\varepsilon$ then $\mathbf{A}$ is invertible and $\mathbf{A}^{-1} \in \mathbf{a d}_{p q}^{s}$.

For the proofs of Propositions 2.1-2.2 see [FJ2] and [FJW]. The only difference is that we have a second index $e \in E\left(\# E=2^{d}-1\right)$ that may affect only the constants.

The following lemma is crucial for the proof of Theorem 2.1 .

Lemma 2.1. If $r \geq 1$ and $M>d+r$, then we have, for $(I, e) \neq\left(J, e^{\prime}\right)$,

$$
\left|a\left(I e, J e^{\prime}\right)\right| \leq C \varepsilon \min \left\{\frac{\ell(I)}{\ell(J)}, \frac{\ell(J)}{\ell(I)}\right\}^{r+d / 2}\left(1+\frac{\left|x_{I}-x_{J}\right|}{\max \{\ell(I), \ell(J)\}}\right)^{-M}
$$

and

$$
|1-a(I e, I e)| \leq C \varepsilon,
$$

where $a\left(I e, J e^{\prime}\right)$ are the entries of the transformation matrix from (2.2) and $C>0$ is independent of $\varepsilon$.

Proof. We consider three different cases:

CASE I: $(I, e) \neq\left(J, e^{\prime}\right),|J| \leq|I|$. We can assume that $|I|=1$ and $x_{I}=0$. The general case follows by a change of variables. We let $e, e^{\prime} \in E$, be fixed and we define $g_{I}^{e}:=\theta_{I}^{e}-\psi_{I}^{e}$. From the orthogonality and the moment condition (A2) of $\Psi$ we have

$$
\begin{aligned}
\left|a\left(I e, J e^{\prime}\right)\right| & =\left|\int_{\mathbb{R}^{d}} g_{I}^{e}(y) \overline{\psi_{J}^{e^{\prime}}(y)} d y\right| \\
& =\left|\int_{\mathbb{R}^{d}}\left[g_{I}^{e}(y)-\sum_{|\eta|<r} \frac{\left(y-x_{J}\right)^{\eta}}{\eta !}\left(g_{I}^{e}\right)^{(\eta)}\left(x_{J}\right)\right] \overline{\psi_{J}^{e^{\prime}}(y)} d y\right| \\
& \leq \int_{\mathbb{R}^{d}}\left|g_{I}^{e}(y)-\sum_{|\eta|<r} \frac{\left(y-x_{J}\right)^{\eta}}{\eta !}\left(g_{I}^{e}\right)^{(\eta)}\left(x_{J}\right)\right|\left|\psi_{J}^{e^{\prime}}(y)\right| d y
\end{aligned}
$$


We will integrate over $A:=\left\{y:\left|y-x_{J}\right| \geq 1\right\}$ and $A^{c}$ separately. For the integral over $A$, we use B1-2 to obtain

$$
\begin{aligned}
& \int_{A}\left|g_{I}^{e}(y)-\sum_{|\eta|<r} \frac{\left(y-x_{J}\right)^{\eta}}{\eta !}\left(g_{I}^{e}\right)^{(\eta)}\left(x_{J}\right)\right|\left|\psi_{J}^{e^{\prime}}(y)\right| d y \\
& \leq C \varepsilon \ell(J)^{-\frac{d}{2}} \int_{A}\left((1+|y|)^{-M}+\frac{\left|y-x_{J}\right|^{r-1}}{\left(1+\left|x_{J}\right|\right)^{M}}\right)\left(1+\frac{\left|y-x_{J}\right|}{\ell(J)}\right)^{-M} d y \\
& =: B_{1}+B_{2},
\end{aligned}
$$

where

$$
B_{1}:=C \varepsilon \ell(J)^{-d / 2} \int_{A}(1+|y|)^{-M}\left(1+\frac{\left|y-x_{J}\right|}{\ell(J)}\right)^{-M} d y
$$

and

$$
B_{2}:=C \varepsilon \ell(J)^{-d / 2} \int_{A}\left|y-x_{J}\right|^{r-1}\left(1+\left|x_{J}\right|\right)^{-M}\left(1+\frac{\left|y-x_{J}\right|}{\ell(J)}\right)^{-M} d y .
$$

For $B_{1}$, we first consider the case where $|y| \leq\left|x_{J}\right| / 2$. In this case, $\left|y-x_{J}\right| \geq\left|x_{J}\right| / 2$ and hence $\frac{\left|y-x_{J}\right|}{\ell(J)} \geq \frac{1+\left|y-x_{J}\right|}{2 \ell(J)} \geq \frac{1+\left|x_{J}\right|}{4 \ell(J)}$. It follows that

$$
\begin{aligned}
& C \varepsilon \ell(J)^{-d / 2} \int_{A \cap\left\{|y| \leq\left|x_{J}\right| / 2\right\}}(1+|y|)^{-M}\left(1+\frac{\left|y-x_{J}\right|}{\ell(J)}\right)^{-M} d y \\
& \leq C \varepsilon \ell(J)^{M-d / 2}\left(1+\left|x_{J}\right|\right)^{-M} \int_{A}(1+|y|)^{-M} d y \\
& \leq C \varepsilon \ell(J)^{M-d / 2}\left(1+\left|x_{J}\right|\right)^{-M} .
\end{aligned}
$$

If $|y|>\left|x_{J}\right| / 2$, then $(1+|y|)^{-M} \leq C\left(1+\left|x_{J}\right|\right)^{-M}$ and hence we have

$$
\begin{aligned}
& C \varepsilon \ell(J)^{-d / 2} \int_{A \cap\left\{|y|>\left|x_{J}\right| / 2\right\}}(1+|y|)^{-M}\left(1+\frac{\left|y-x_{J}\right|}{\ell(J)}\right)^{-M} d y \\
& \leq C \varepsilon \ell(J)^{-d / 2}\left(1+\left|x_{J}\right|\right)^{-M} \int_{A \cap\left\{|y|>\left|x_{J}\right| / 2\right\}}\left(1+\frac{\left|y-x_{J}\right|}{\ell(J)}\right)^{-M} d y \\
& \leq C \varepsilon \ell(J)^{-d / 2}\left(1+\left|x_{J}\right|\right)^{-M} \int_{\left|y-x_{J}\right|>1}\left(\frac{1+\left|y-x_{J}\right|}{\ell(J)}\right)^{-M} d y \\
& \leq C \varepsilon \ell(J)^{M-d / 2}\left(1+\left|x_{J}\right|\right)^{-M} .
\end{aligned}
$$

For $B_{2}$, using that $\left|y-x_{J}\right| \geq 1$, we have

$$
\begin{aligned}
B_{2} & \leq C \varepsilon\left(1+\left|x_{J}\right|\right)^{-M} \ell(J)^{r-1-d / 2} \int_{A}\left(1+\frac{\left|y-x_{J}\right|}{\ell(J)}\right)^{-M+r-1} d y \\
& \leq C \varepsilon\left(1+\left|x_{J}\right|\right)^{-M} \ell(J)^{r-1-d / 2} \int_{A}\left(\frac{1+\left|y-x_{J}\right|}{\ell(J)}\right)^{-M+r-1} d y \\
& \leq C \varepsilon\left(1+\left|x_{J}\right|\right)^{-M} \ell(J)^{M-d / 2} .
\end{aligned}
$$

Since $\ell(J) \leq 1$ and $M-d / 2>r+d / 2$, from (2.5) - (2.7), we find

$$
\int_{A}\left|g_{I}^{e}(y)-\sum_{|\eta|<r} \frac{\left(y-x_{J}\right)^{\eta}}{\eta !}\left(g_{I}^{e}\right)^{(\eta)}\left(x_{J}\right)\right|\left|\psi_{J}^{e^{\prime}}(y)\right| d y \leq C \ell(J)^{r+d / 2}\left(1+\left|x_{J}\right|\right)^{-M} .
$$


Next, we estimate the integral over $A^{c}=\left\{y:\left|y-x_{J}\right|<1\right\}$. Using B1-2, we get

$$
\begin{aligned}
& \int_{A^{c}}\left|g_{I}^{e}(y)-\sum_{|\eta|<r} \frac{\left(y-x_{J}\right)^{\eta}}{\eta !}\left(g_{I}^{e}\right)^{(\eta)}\left(x_{J}\right)\right|\left|\psi_{J}^{e^{\prime}}(y)\right| d y \\
= & \int_{A^{c}}\left|\sum_{|\eta|=r} \int_{0}^{1} \frac{\left(y-x_{J}\right)^{\eta}}{\eta !}\left(g_{I}^{e}\right)^{(\eta)}\left(x_{J}+t\left(y-x_{J}\right)\right) t^{r-1} d t\right|\left|\psi_{J}^{e^{\prime}}(y)\right| d y \\
\leq & C \varepsilon \ell(J)^{-d / 2} \int_{A^{c}}\left|y-x_{J}\right|^{r} \sup _{z}(1+|z|)^{-M}\left(1+\frac{\left|y-x_{J}\right|}{\ell(J)}\right)^{-M} d y \\
\leq & C \varepsilon \ell(J)^{r-d / 2} \int_{A^{c}} \sup _{z}(1+|z|)^{-M}\left(1+\frac{\left|y-x_{J}\right|}{\ell(J)}\right)^{-M+r} d y,
\end{aligned}
$$

where the supremum is taken over all $z$ lying on the line segment joining $x_{J}$ and $y$. It follows that $\left|x_{J}\right| \leq\left|z-x_{J}\right|+|z| \leq 1+|z|$ which implies

$$
\sup _{z}(1+|z|)^{-M} \leq C\left(1+\left|x_{J}\right|\right)^{-M} \text {. }
$$

Using this estimate in (2.8), we finally obtain

$$
\begin{aligned}
& \int_{A^{c}}\left|g_{I}^{e}(y)-\sum_{|\eta|<r} \frac{\left(y-x_{J}\right)^{\eta}}{\eta !}\left(g_{I}^{e}\right)^{(\eta)}\left(x_{J}\right)\right|\left|\psi_{J}^{e^{\prime}}(y)\right| d y \\
& \leq C \varepsilon \ell(J)^{r-d / 2}\left(1+\left|x_{J}\right|\right)^{-M} \int_{A^{c}}\left(1+\frac{\left|y-x_{J}\right|}{\ell(J)}\right)^{-M+r} d y \\
& \leq C \varepsilon \ell(J)^{r+d / 2}\left(1+\left|x_{J}\right|\right)^{-M} .
\end{aligned}
$$

CASE II: $(I, e) \neq\left(J, e^{\prime}\right),|I|<|J|$. Again we may assume that $|J|=1$ and $x_{J}=0$. We fix $e, e^{\prime} \in E$, and define $g_{I}^{e}:=\theta_{I}^{e}-\psi_{I}^{e}$. From the orthogonality and the moment condition B3, we have

$$
\begin{aligned}
\left|a\left(I e, J e^{\prime}\right)\right| & =\left|\int_{\mathbb{R}^{d}} g_{I}^{e}(y) \overline{\psi_{J}^{e^{\prime}}(y)} d y\right| \\
& \left.=\mid \int_{\mathbb{R}^{d}} g_{I}^{e}(y) \overline{\psi_{J}^{e}(y)}-\sum_{|\eta|<r} \frac{\left(y-x_{I}\right)^{\eta}}{\eta !}\left(\overline{\psi_{J}^{e}}\right)^{(\eta)}\left(x_{I}\right)\right] d y \mid \\
& \leq \int_{\mathbb{R}^{d}}\left|g_{I}^{e}(y)\right|\left|\psi_{J}^{e}(y)-\sum_{|\eta|<r} \frac{\left(y-x_{I}\right)^{\eta}}{\eta !}\left(\psi_{J}^{e}\right)^{(\eta)}\left(x_{I}\right)\right| d y .
\end{aligned}
$$

The result now follows as in the previous case by interchanging the roles of $g^{e}$ and $\psi^{e}$.

CASE III: $(I, e)=\left(J, e^{\prime}\right)$. Using the orthonormality of $\Psi$, we get

$$
a(I e, I e)=1+\int_{\mathbb{R}^{d}}\left(\theta_{I}^{e}(y)-\psi_{I}^{e}(y)\right) \overline{\psi_{I}^{e}(y)} d y
$$

which gives

$$
|a(I e, I e)-1|=\left|\int_{\mathbb{R}^{d}}\left(\theta_{I}^{e}(y)-\psi_{I}^{e}(y)\right) \overline{\psi_{I}^{e}(y)} d y\right|
$$

and the proof follows identically as in the first case.

Proof of Theorem [2.1. We will prove only part (a) of the theorem. The proof of part (b) is the same and will be omitted. 
Let $s \in \mathbb{R}, 0<p<\infty, 0<q \leq \infty, \mathcal{J}:=d / \min \{1, p, q\}, r>\max \{\mathcal{J}-d-s, s\}$, and $M>\max \{\mathcal{J}, d+r\}$. By Lemma 2.1], it is immediate that there exists $\delta>0$ such that

$$
\left|a\left(I e, J e^{\prime}\right)\right| \leq C \varepsilon \omega_{\delta}(I, J), \quad(I, e),\left(J, e^{\prime}\right) \in D \times E,
$$

and

$$
|1-a(I e, I e)| \leq C \varepsilon, \quad(I, e) \in D \times E .
$$

Therefore, $\mathbf{A}_{\varepsilon} \in \mathbf{a d}_{p q}^{s}$ and $\left\|\mathbf{I}-\mathbf{A}_{\varepsilon}\right\|_{\delta} \leq C \varepsilon$. Applying Proposition 2.2, we obtain that for sufficiently small $\varepsilon>0$ the matrix $\mathbf{A}_{\varepsilon}$ is invertible and $\mathbf{A}_{\varepsilon}^{-1} \in \mathbf{a d}_{p q}^{s}$. Now, Proposition 2.1 implies that $\mathbf{A}_{\varepsilon}$ and $\mathbf{A}_{\varepsilon}^{-1}$ are both bounded on $\dot{f}_{p q}^{s}$.

Estimate (2.3) from Lemma 2.1 is symmetric about $I$ and $J$. Therefore, the above holds for $\mathbf{A}_{\varepsilon}^{T}$ and $\left(\mathbf{A}_{\varepsilon}^{T}\right)^{-1}$ instead of $\mathbf{A}_{\varepsilon}$ and $\mathbf{A}_{\varepsilon}^{-1}$, respectively.

\section{New bases for homogeneous Triebel-Lizorkin and Besov spaces}

Our goal in this section is to prove the following theorems:

Theorem 3.1. Let $s \in \mathbb{R}, 0<p<\infty, 0<q \leq \infty$, and $\mathcal{J}:=d / \min \{1, p, q\}$. If $r>\max \{\mathcal{J}-d-s, s\}$ and $M>\max \{\mathcal{J}, d+r\}$, then for sufficiently small $\varepsilon$ the new system $B$ is an unconditional basis for the space $\dot{F}_{p q}^{s}$. That is, for every $f \in \dot{F}_{p q}^{s}$ there exists a unique sequence $d:=\left(d_{I, e}\right)$ such that $f=\sum_{I, e} d_{I e} \theta_{I}^{e}$ in $\mathcal{S}^{\prime} / \mathcal{P}$ (and in $\dot{F}_{p q}^{s}$ if $\left.q \neq \infty\right)$ and

$$
\|f\|_{\dot{F}_{p q}^{s}} \approx\left\|\left(\sum_{I, e}\left(|I|^{-s / d}\left|d_{I e} \lambda_{I}\right|\right)^{q}\right)^{1 / q}\right\|_{L_{p}} .
$$

Theorem 3.2. Let $s \in \mathbb{R}, 0<p \leq \infty, 0<q \leq \infty$, and $\mathcal{J}:=d / \min \{1, p\}$. If $r>\max \{\mathcal{J}-d-s, s\}$ and $M>\max \{\mathcal{J}, d+r\}$, then for sufficiently small $\varepsilon$ the new system $B$ is an unconditional basis for the space $\dot{B}_{p q}^{s}$. That is, for every $f \in \dot{B}_{p q}^{s}$ there exists a unique sequence $d:=\left(d_{I, e}\right)$ such that $f=\sum_{I, e} d_{I e} \theta_{I}^{e}$ in $\mathcal{S}^{\prime} / \mathcal{P}$ (and in $\dot{B}_{p q}^{s}$ if $\left.p, q \neq \infty\right)$ and

$$
\|f\|_{\dot{B}_{p q}^{s}} \approx\left(\sum_{e \in E} \sum_{m \in \mathbb{Z}}\left(\sum_{I \in D_{m}}\left(|I|^{-s / d+1 / p-1 / 2}\left|d_{I e}\right|\right)^{p}\right)^{q / p}\right)^{1 / q} .
$$

For the proofs of Theorems 3.1,3.2, we will need the following two lemmas.

Lemma 3.1. Let $s \in \mathbb{R}, 0<p<\infty, 0<q \leq \infty$, and $\mathcal{J}:=d / \min \{1, p, q\}$. If $r>\max \{\mathcal{J}-d-s, s\}, M>\max \{\mathcal{J}, d+r\}$, and $\left\{\theta_{I}^{e}: I \in D, e \in E\right\}$ is a family of functions satisfying B2-3, then for every $d:=\left(d_{I e}\right) \in \dot{f}_{p q}^{s}$ the series $\sum_{I, e} d_{I e} \theta_{I}^{e}$ converges in $\mathcal{S}^{\prime} / \mathcal{P}$ (and in $\dot{F}_{p q}^{s}$ for $q \neq \infty$ ) and

$$
\left\|\sum_{I, e} d_{I e} \theta_{I}^{e}\right\|_{\dot{F}_{p q}^{s}} \leq C\|d\|_{\dot{f}_{p q}^{s}} .
$$

Proof. Let $\eta \in S_{\infty}$. Exactly as in the proof of Lemma 2.1, one can show that

$$
\left|\left\langle\theta_{I}^{e}, \eta\right\rangle\right| \leq C \min \left\{\ell(I), \ell(I)^{-1}\right\}^{r+d / 2}\left(1+\frac{\left|x_{I}\right|}{\max \{\ell(I), 1\}}\right)^{-M}, \quad(I, e) \in D \times E .
$$


From this, we find

$$
\begin{aligned}
\left|\sum_{I, e} d_{I e}\left\langle\theta_{I}^{e}, \eta\right\rangle\right| & \leq \sum_{I, e}\left|d_{I e}\right|\left|\left\langle\theta_{I}^{e}, \eta\right\rangle\right| \\
& \leq C \sum_{I, e}\left|d_{I e}\right| \min \left\{\ell(I), \ell(I)^{-1}\right\}^{r+d / 2}\left(1+\frac{\left|x_{I}\right|}{\max \{\ell(I), 1\}}\right)^{-M} \\
& =\sum_{\substack{|I|>1 \\
e}}+\sum_{|I| \leq 1}=: \sigma_{1}+\sigma_{2} .
\end{aligned}
$$

Since $d \in \dot{f}_{p q}^{s}$, then $\left|d_{I e}\right| \leq C|I|^{s / d+1 / 2-1 / p},(I, e) \in D \times E$, and hence

$$
\begin{aligned}
\sigma_{1} & \leq C \sum_{|I|>1}|I|^{s / d+1 / 2-1 / p-r / d-1 / 2}\left(1+\left|x_{I}\right| / \ell(I)\right)^{-M} \\
& \leq C \sum_{\mu \geq 0} 2^{\mu(s-r-d / p)} \sum_{\substack{I \in D_{\mu} \\
e}}\left(1+\left|x_{I}\right| / \ell(I)\right)^{-M} \\
& \leq C \sum_{\mu \geq 0} 2^{\mu(s-r-d / p)}<\infty .
\end{aligned}
$$

To estimate $\sigma_{2}$ we will use the maximal operator $M_{t}$ defined by

$$
M_{t}(f)(x):=\left(\sup _{Q \ni x}|Q|^{-1} \int_{Q}|f(y)|^{t} d y\right)^{1 / t}
$$

where the sup is taken over all cubes $Q$ (containing $x$ ) with sides parallel to the axes, and $t$ is selected so that $0<t<\min \{1, p, q\}, M>d / t$, and $r>d / t-d-s$. We now use Lemma 6.1 from Appendix to obtain, for every $x$ in the unit cube $I_{0}$,

$$
\begin{aligned}
\sigma_{2} & :=C \sum_{|I| \leq 1}|I|^{\frac{r}{d}+\frac{1}{2}}\left|d_{I e}\right|\left(1+\left|x_{I}\right|\right)^{-M} \leq C \sum_{\mu \geq 0} 2^{-\mu(r+d / 2)} \sum_{\substack{I \in D_{\mu} \\
e}}\left|d_{I e}\right|\left(1+\left|x_{I}\right|\right)^{-M} \\
& \leq C \sum_{\mu \geq 0} 2^{-\mu(r+d / 2-d / t)} M_{t}\left(\sum_{\substack{I \in D_{\mu} \\
e}}\left|d_{I e}\right| \chi_{I}\right)(x) .
\end{aligned}
$$

We denote $\tilde{\lambda}_{I}:=|I|^{-s / d-1 / 2} \chi_{I}$. Using that $r>d / t-d-s$, we have, for $x \in I_{0}$,

$$
\begin{aligned}
\sigma_{2} & \leq C \sum_{\mu \geq 0} 2^{-\mu(r+s+d-d / t)} M_{t}\left(\sum_{I \in D_{\mu}}\left|d_{I e}\right| \tilde{\lambda}_{I}\right)(x) \leq C \sup _{\mu \geq 0} M_{t}\left(\sum_{I \in D_{\mu}}\left|d_{I e}\right| \tilde{\lambda}_{I}\right)(x) \\
& \leq C\left(\sum_{\mu \geq 0}\left[M_{t}\left(\sum_{\substack{I \in D_{\mu} \\
e}}\left|d_{I e}\right| \tilde{\lambda}_{I}\right)(x)\right]^{q}\right)^{1 / q} .
\end{aligned}
$$

We now take the $L_{p}\left(I_{0}\right)$ norm and use the Fefferman-Stein maximal inequality (see Proposition 6.3) to obtain

$$
\sigma_{2} \leq C\left\|\left(\sum_{\mu \geq 0}\left[M_{t}\left(\sum_{I \in D_{\mu}}\left|d_{I e}\right| \tilde{\lambda}_{I}\right)\right]^{q}\right)^{1 / q}\right\|_{L_{p}\left(I_{0}\right)} \leq C\|d\|_{\dot{f}_{p q}^{s}}<\infty .
$$

Therefore, the series $f:=\sum_{I, e} d_{I e} \theta_{I}^{e}$ converges in $\mathcal{S}^{\prime} / \mathcal{P}$.

It remains to prove (3.3). From the above, we find

$$
c_{I e}:=\left\langle f, \psi_{I}^{e}\right\rangle=\sum_{J, e^{\prime}} d_{J e^{\prime}}\left\langle\theta_{J}^{e^{\prime}}, \psi_{I}^{e}\right\rangle=\sum_{J, e^{\prime}} d_{J e^{\prime}} a\left(J e^{\prime}, I e\right), \quad(I, e) \in D \times E .
$$


Denoting $c:=\left(c_{I e}\right)$, we have

$$
c=\mathbf{A}^{T} d
$$

where $\mathbf{A}:=\mathbf{A}_{\varepsilon}$ is the transformation matrix from (2.2).

By Theorem 2.1, $\mathbf{A}^{T}$ is bounded on $\dot{f}_{p q}^{s}$. Therefore, using (1.2), we have

$$
\left\|\sum_{I, e} c_{I e} \psi_{I}^{e}\right\|_{\dot{F}_{p q}^{s}} \approx\|c\|_{\dot{f}_{p q}^{s}}=\left\|\mathbf{A}^{T} d\right\|_{\dot{f}_{p q}^{s}} \leq C\|d\|_{\dot{f}_{p q}^{s}} .
$$

Finally, we note that once (3.3) has been established, it follows that for $q \neq \infty$ the series $\sum_{I, e} d_{I e} \theta_{I}^{e}$ converges in the sense of $\dot{F}_{p q}^{s}$, since its tail $\sum_{|I| \geq N, e} d_{I e} \theta_{I}^{e}$ converges strongly to 0 , as $N \rightarrow \infty$.

Lemma 3.2. Let $s \in \mathbb{R}, 0<p \leq \infty, 0<q \leq \infty$, and $\mathcal{J}:=d / \min \{1, p\}$. If $r>\max \{\mathcal{J}-d-s, s\}, M>\max \{\mathcal{J}, d+r\}$, and $\left\{\theta_{I}^{e}: I \in D, e \in E\right\}$ satisfies $\boldsymbol{B 2 - 3 , ~ t h e n ~ f o r ~ e v e r y ~} d:=\left(d_{I e}\right) \in \dot{b}_{p q}^{s}$ the series $\sum_{I, e} d_{I e} \theta_{I}^{e}$ converges in $\mathcal{S}^{\prime} / \mathcal{P}$ (and in $\dot{B}_{p q}^{s}$ for $\left.p, q \neq \infty\right)$ and

$$
\left\|\sum_{I, e} d_{I e} \theta_{I}^{e}\right\|_{\dot{B}_{p q}^{s}} \leq C\|d\|_{\dot{b}_{p q}}
$$

Proof. The proof of (3.6) is identical to the one of (3.3) since under our assumptions the matrix $\mathbf{A}^{T}$ is bounded on $\dot{b}_{p q}^{s}$. Therefore, we need only establish that the series $\sum_{I, e} d_{I e} \theta_{I}^{e}$ converges in $\mathcal{S}^{\prime} / \mathcal{P}$. For this, we note that for every $\eta \in S_{\infty}$

$$
\begin{aligned}
\left|\sum_{I, e} d_{I e}\left\langle\theta_{I}^{e}, \eta\right\rangle\right| & \leq \sum_{I, e}\left|d_{I e}\right|\left|\left\langle\theta_{I}^{e}, \eta\right\rangle\right| \\
& \leq \sum_{I, e}\left|d_{I e}\right| \min \left\{\ell(I), \ell(I)^{-1}\right\}^{r+d / 2}\left(1+\frac{\left|x_{I}\right|}{\max \{\ell(I), 1\}}\right)^{-M} \\
& =\sum_{|I|>1}+\sum_{|I| \leq 1}=: \sigma_{1}+\sigma_{2},
\end{aligned}
$$

where we used (3.4). Since $d \in \dot{b}_{p q}^{s}$, then $\left|d_{I e}\right| \leq C|I|^{s / d+1 / 2-1 / p},(I, e) \in D \times E$, and the proof of Lemma 3.1 gives us immediately that $\sigma_{1}<\infty$.

For $\sigma_{2}$, we will consider two cases.

CASE I: $0<p \leq 1$. We have

$$
\begin{aligned}
\sigma_{2} & =\sum_{|I| \leq 1}|I|^{r / d+1 / 2}\left|d_{I e}\right|\left(1+\left|x_{I}\right|\right)^{-M} \\
& \leq \sum_{m \geq 0} 2^{-m(r+s+d-d / p)} \sum_{I \in D_{e}}|I|^{-s / d-1 / 2+1 / p}\left|d_{I e}\right| \\
& \leq \sup _{m \geq 0}\left(\sum_{I \in D_{m}}\left(|I|^{-s / d-1 / 2+1 / p}\left|d_{I e}\right|\right)^{p}\right)^{1 / p} \leq C\|d\|_{\dot{b}_{p q}^{s}}<\infty
\end{aligned}
$$

where we used the inequality: $\sum\left|x_{j}\right| \leq\left(\sum\left|x_{j}\right|^{p}\right)^{1 / p}, 0<p \leq 1$.

CASE II: $p>1$. We use Hölder's inequality and the obvious inequality

$$
\sum_{I \in D_{m}}\left(1+\left|x_{I}\right|\right)^{-M^{\diamond}} \leq C|I|^{-1}, \quad I \in D_{m}, \quad m \geq 0, \quad M^{\diamond}>d
$$


to obtain

$$
\begin{aligned}
\sigma_{2} & =\sum_{|I| \leq 1}|I|^{r / d+1 / 2}\left|d_{I e}\right|\left(1+\left|x_{I}\right|\right)^{-M} \\
& \leq \sum_{m \geq 0} 2^{-m(r+d / 2)}\left(\sum_{\substack{I \in D_{m} \\
e}}\left|d_{I e}\right|^{p}\right)^{1 / p}\left(\sum_{\substack{I \in D_{m} \\
e}}\left(1+\left|x_{I}\right|\right)^{-M p^{\prime}}\right)^{1 / p^{\prime}} \\
& \leq \sum_{m \geq 0} 2^{-m\left(r+d / 2-d / p^{\prime}\right)}\left(\sum_{I \in D_{m}}\left|d_{I e}\right|^{p}\right)^{1 / p} \\
& =\sum_{m \geq 0} 2^{-m(r+s)}\left(\sum_{I \in D_{m}}\left(|I|^{-s / d-1 / 2+1 / p}\left|d_{I e}\right|\right)^{p}\right)^{1 / p} \leq C\|d\|_{\dot{b}_{p q}^{s}}<\infty,
\end{aligned}
$$

where we used that $1 / p^{\prime}:=1-1 / p$.

Corollary 3.1. Let $s, p, q, r$, and $M$ be as in Lemma 3.1 in the F-space case and as in Lemma 3.2 in the B-space case. Let $\mathbf{A}:=\left(a\left(I e, J e^{\prime}\right)\right)$ and $\mathbf{A}^{-1}=:\left(b\left(I e, J e^{\prime}\right)\right)$ be the transformation matrix 2.2 and its inverse from Theorem 2.1. Then, we have

$$
\theta_{I}^{e}=\sum_{J, e^{\prime}} a\left(I e, J e^{\prime}\right) \psi_{J}^{e^{\prime}}, \quad \psi_{I}^{e}=\sum_{J, e^{\prime}} b\left(I e, J e^{\prime}\right) \theta_{J}^{e \prime}, \quad(I, e) \in D \times E,
$$

where the convergence is considered in $\mathcal{S}^{\prime} / \mathcal{P}$ as well as in $\dot{F}_{p q}^{s},\left(\dot{B}_{p q}^{s}\right)$ for $p, q \neq \infty$. Proof. For a fixed $(I, e) \in D \times E$, we define the sequence $\delta^{I e}:=\left(\delta_{J, e^{\prime}}^{I e}\right)$ by

$$
\delta_{J e^{\prime}}^{I e}:= \begin{cases}1, & (I, e)=\left(J, e^{\prime}\right) \\ 0, & (I, e) \neq\left(J, e^{\prime}\right) .\end{cases}
$$

Then, $\left(a\left(I e, J e^{\prime}\right)_{J e^{\prime}}\right)=\mathbf{A}^{T} \delta^{I e}$ and $\left(b\left(I e, J e^{\prime}\right)_{J e^{\prime}}\right)=\left(\mathbf{A}^{T}\right)^{-1} \delta^{I e}$. By Theorem $2.1 \mathbf{A}^{T}$ and $\left(\mathbf{A}^{T}\right)^{-1}$ are bounded on $\dot{f}_{p q}^{s}\left(\dot{b}_{p q}^{s}\right)$ and consequently $\left(a\left(I e, J e^{\prime}\right)_{J e^{\prime}}\right)$, $\left(b\left(I e, J e^{\prime}\right)_{J e^{\prime}}\right) \in \dot{f}_{p q}^{s}\left(\dot{b}_{p q}^{s}\right)$. As far as the convergence of first series is concerned, the result follows immediately from the wavelet characterization of $\dot{F}_{p q}^{s}\left(\dot{B}_{p q}^{s}\right)$ and Lemma 3.1 (3.2). For the second series we note that by Lemma 3.1 (or 3.2) the series converges in $\mathcal{S}^{\prime} / \mathcal{P}$ as well as in $\dot{F}_{p q}^{s}\left(\dot{B}_{p q}^{s}\right)$ and that for each $\left(\Delta, e^{\prime \prime}\right) \in D \times E$,

$$
\left\langle\sum_{J, e^{\prime}} b\left(I e, J e^{\prime}\right) \theta_{J}^{e \prime}, \psi_{\Delta}^{e^{\prime \prime}}\right\rangle=\sum_{J, e^{\prime}} b\left(I e, J e^{\prime}\right) a\left(J e^{\prime}, \Delta e^{\prime \prime}\right)= \begin{cases}1, & (I, e)=\left(\Delta, e^{\prime \prime}\right), \\ 0, & (I, e) \neq\left(\Delta, e^{\prime \prime}\right) .\end{cases}
$$

From the completeness of the wavelet basis we get $\psi_{I}^{e}=\sum_{J, e^{\prime}} b\left(I e, J e^{\prime}\right) \theta_{J}^{e \prime}$.

Proof of Theorem [3.1] We will first prove that for every $f \in \dot{F}_{p q}^{s}$ there exist coefficients $d:=\left(d_{I e}\right)$ such that $f=\sum_{I, e} d_{I e} \theta_{I}^{e}$ in $\mathcal{S}^{\prime} / \mathcal{P}$. Since $f \in \dot{F}_{p q}^{s}$, from the wavelet decomposition of $f$, we have

$$
f=\sum_{I, e} c_{I e} \psi_{I}^{e}, \quad c_{I e}:=c_{I e}(f):=\left\langle f, \psi_{I}^{e}\right\rangle,
$$

in the sense of $\mathcal{S}^{\prime} / \mathcal{P}$, where $c:=\left(c_{I e}\right) \in \dot{f}_{p q}^{s}$ and

From Corollary 3.1 we have

$$
\|f\|_{\dot{F}_{p q}^{s}} \approx\|c\|_{\dot{f}_{p q}^{s}} \text {. }
$$

$$
\psi_{I}^{e}=\sum_{J, e^{\prime}} b\left(I e, J e^{\prime}\right) \theta_{J}^{e \prime}, \quad(I, e) \in D \times E,
$$


in $\mathcal{S}^{\prime} / \mathcal{P}$. Therefore,

$$
\begin{aligned}
f & =\sum_{I, e} c_{I e} \psi_{I}^{e}=\sum_{I, e} c_{I e} \sum_{J, e^{\prime}} b\left(I e, J e^{\prime}\right) \theta_{J}^{e \prime} \\
& =\sum_{J, e^{\prime}} \sum_{I, e} b\left(I e, J e^{\prime}\right) c_{I e} \theta_{J}^{e \prime} \\
& =\sum_{J, e^{\prime}} d_{J e^{\prime}} \theta_{J}^{e \prime},
\end{aligned}
$$

where all identities above are considered in the distributional sense and $d_{J e^{\prime}}:=$ $\sum_{I, e} b\left(I e, J e^{\prime}\right) c_{I e}$. To justify the third equality, we note that the assumptions of the theorem guarantee that the matrix $\mathbf{A}^{-1}=\left(b\left(I e, J e^{\prime}\right)\right)$ is bounded on $\dot{f}_{p q}^{s}$. Since $c \in \dot{f}_{p q}^{s}$, the sequence $a:=\left(a_{J e^{\prime}}\right):=\left(\sum_{I, e}\left|b\left(I e, J e^{\prime}\right)\right|\left|c_{I e}\right|\right)_{J, e^{\prime}}$ belongs in $\dot{f}_{p q}^{s}$. At last for every $\eta \in \mathcal{S}_{\infty}$

$$
\sum_{J, e^{\prime}}\left|a_{J e^{\prime}}\right|\left|\left\langle\theta_{J}^{e \prime}, \eta\right\rangle\right|<\infty
$$

as it follows from the proof of Lemma 3.1 Therefore, the order of summation can be interchanged.

Next, we will prove the norm equivalence (3.1) which also guarantees the uniqueness of the coefficients. Since $\mathbf{A}^{T},\left(\mathbf{A}^{T}\right)^{-1}$ are bounded on $\dot{f}_{p q}^{s}$, by Theorem 2.1, and

$$
c_{I e}=\sum_{J, e^{\prime}} a\left(J e^{\prime}, I e\right) d_{J e^{\prime}}, \quad d_{I e}=\sum_{J, e^{\prime}} b\left(J e^{\prime}, I e\right) c_{J e^{\prime}}
$$

we have

$$
c=\mathbf{A}^{T} d, \quad d=\left(\mathbf{A}^{T}\right)^{-1} c .
$$

Therefore,

$$
\|c\|_{\dot{f}_{p q}^{s}}=\left\|\mathbf{A}^{T} d\right\|_{\dot{f}_{p q}^{s}} \leq C_{1}\|d\|_{\dot{f}_{p q}^{s}}=C_{1}\left\|\left(\mathbf{A}^{T}\right)^{-1} c\right\|_{\dot{f}_{p q}^{s}} \leq C_{2}\|c\|_{\dot{f}_{p q}^{s}}
$$

which is the desired condition (3.1) since $\|c\|_{\dot{f}_{p q}^{s}} \approx\|f\|_{\dot{F}_{p q}^{s}}$. This concludes the proof of the theorem.

Proof of Theorem 3.2. The proof follows the footsteps of the one of Theorem 3.1 It is sufficient to prove that for every $f \in \dot{B}_{p q}^{s}$ there exist coefficients $d=\left(d_{I e}\right)$ such that $f=\sum_{I, e} d_{I e} \theta_{I}^{e}$ in $\mathcal{S}^{\prime} / \mathcal{P}$, and then to establish the norm equivalence (3.2).

Since $f \in \dot{B}_{p q}^{s}$, from the wavelet decomposition of $f$, we have

$$
f=\sum_{I, e} c_{I e} \psi_{I}^{e}, \quad c_{I e}:=c_{I e}(f):=\left\langle f, \psi_{I}^{e}\right\rangle,
$$

in the sense of $\mathcal{S}^{\prime} / \mathcal{P}$, where $c:=\left(c_{I e}\right) \in \dot{b}_{p q}^{s}$ and

$$
\|f\|_{\dot{B}_{p q}^{s}} \approx\|c\|_{\dot{b}_{p q}^{s}} \text {. }
$$

Employing Corollary 3.1, we get that for every $I \in D, e \in E$,

$$
\psi_{I}^{e}=\sum_{J, e^{\prime}} b\left(I e, J e^{\prime}\right) \theta_{J}^{e \prime},
$$


in $\mathcal{S}^{\prime} / \mathcal{P}$. It follows that

$$
\begin{aligned}
f & =\sum_{I, e} c_{I e} \psi_{I}^{e}=\sum_{I, e} c_{I e} \sum_{J, e^{\prime}} b\left(I e, J e^{\prime}\right) \theta_{J}^{e \prime} \\
& =\sum_{J, e^{\prime}} \sum_{I, e} b\left(I e, J e^{\prime}\right) c_{I e} \theta_{J}^{e \prime} \\
& =\sum_{J, e^{\prime}} d_{J e^{\prime}} \theta_{J}^{e \prime}
\end{aligned}
$$

where all equalities above are considered in the distributional sense and $d_{J e^{\prime}}:=$ $\sum_{I, e} b\left(I e, J e^{\prime}\right) c_{I e}$. The third equality can be justified by the fact that the matrix $\left(\mathbf{A}^{T}\right)^{-1}$ is bounded on $\dot{b}_{p q}^{s}$, by Theorem 2.1. Since $c \in \dot{b}_{p q}^{s}$, the sequence $a:=$ $\left(a_{J e^{\prime}}\right):=\left(\sum_{I, e}\left|b\left(I e, J e^{\prime}\right)\right|\left|c_{I e}\right|\right)_{J, e^{\prime}}$ belongs in $\dot{b}_{p q}^{s}$. At last for every $\eta \in \mathcal{S}_{\infty}$

$$
\sum_{J, e^{\prime}}\left|a_{J e^{\prime}}\right|\left|\left\langle\theta_{J}^{e \prime}, \eta\right\rangle\right|<\infty
$$

as it follows from the proof of Lemma 3.2 Therefore, the order of summation can be interchanged. that

To prove (3.1) we note that $\mathbf{A}^{T},\left(\mathbf{A}^{T}\right)^{-1}$ are bounded on $\dot{b}_{p q}^{s}$ (Theorem 2.1) and

$$
c=\mathbf{A}^{T} d, \quad d=\left(\mathbf{A}^{T}\right)^{-1} c .
$$

Therefore,

$$
\|c\|_{\dot{b}_{p q}^{s}}=\left\|\mathbf{A}^{T} d\right\|_{\dot{b}_{p q}^{s}} \leq C_{1}\|d\|_{\dot{b}_{p q}^{s}}=C_{1}\left\|\left(\mathbf{A}^{T}\right)^{-1} c\right\|_{\dot{b}_{p q}^{s}} \leq C_{2}\|c\|_{\dot{b}_{p q}^{s}}
$$

Using now (3.8) the result follows.

\section{EXAmples OF NeW BASES}

In the previous sections, we proved that if a family of functions $\left\{\theta^{e}: e \in E\right\}$ approximates a suitable wavelet set $\left\{\psi^{e}: e \in E\right\}$ in the sense that, for sufficiently small $\varepsilon>0$, (1.4) and (1.5) hold, then

$$
B:=\left\{\theta_{I}^{e}: I \in D, e \in E\right\}
$$

constitutes an unconditional basis for various types of spaces, depending on the size of $M$ and $r$. Our goal now is to show how this technique can be used for the construction of bases $B:=\left\{\theta_{I}^{e}: I \in D, e \in E\right\}$ with $\theta^{e}$ a linear combination of a "small" number of shifts and dilates of a single function. Next, we give two constructions. The first construction is more general but not so simple and constructive as the second one.

- General construction of bases from shifts and dilates of a single function.

Let $\Phi_{n}, n=1,2, \ldots$, be a family of functions in $C^{r+1}\left(\mathbb{R}^{d}\right)(r \geq 1)$ which satisfy the following conditions:

C1. There exist $M^{\diamond}>d+r, \mu>0, \eta>0$, and a constant $C>0$ such that

$$
\left|\Phi_{n}^{(\alpha)}(x)\right| \leq C \frac{n^{|\alpha| \mu+d}}{(1+n|x|)^{M^{\diamond}}}, \quad|\alpha| \leq r+1, \quad n=1,2, \ldots
$$

C2.

$$
\int_{\mathbb{R}^{d}} \Phi_{n}(x) d x=1, \quad n=1,2, \ldots
$$


The most interesting examples of $\Phi_{n}$ 's that satisfy these conditions are the family of exponentials $\Phi_{n}(\cdot):=C_{1} n^{d} e^{-n^{2}|\cdot|^{2}}$ and the rational functions $\Phi_{n}(\cdot):=$ $C_{2} n^{d}\left(1+n^{2}|\cdot|^{2}\right)^{-N}, N>M^{\diamond} / 2$, and $N>d / 2$, where the constants $C_{1}$ and $C_{2}$ are chosen so that $\mathbf{C 2}$ holds.

More general examples of $\Phi$ 's can be constructed as follows: Let $\Phi(\cdot):=\varphi\left(|\cdot|^{2}\right)$ be a radial function such that $\int \Phi(x) d x=1$, where $\varphi$ is even, $\varphi \in C^{r+1}$, and $\left|\varphi^{(\nu)}(t)\right| \leq C(1+|t|)^{-M^{\diamond}}, t \in \mathbb{R}, \nu=0, \ldots, r+1$, with $M^{\diamond} \geq d+r$. Evidently, $\Phi_{n}(\cdot):=n^{d} \varphi(n \cdot)$ satisfy $\mathbf{C 1 - 2 .}$

We denote

$$
\Theta_{K}:=\left\{\theta: \theta(\cdot)=\sum_{j=1}^{\mu} a_{j} \Phi_{n}\left(\cdot+b_{j}\right), \quad \mu n \leq K\right\} .
$$

The next theorem will enable us to construct bases $B:=\left\{\theta_{I}^{e}: I \in D, e \in E\right\}$ with $\theta_{I}^{e} \in \Theta_{K}, K$ fixed.

In what follows, we consider Meyer's wavelet set $\Psi:=\left\{\psi^{e}: e \in E\right\}$ of $d$-fold tensor products of the scaling function and the wavelet (see [M]). Obviously, each $\psi^{e} \in \mathcal{S}$ and, therefore, $\psi^{e}$ satisfies the following assumptions for any choice of the parameters:

$\mathbf{A 1} 1^{\prime} \cdot \psi^{e} \in C^{r+1}\left(\mathbb{R}^{d}\right)$ and

$$
\left|\left(\psi^{e}\right)^{(\alpha)}(x)\right| \leq C(1+|x|)^{-M^{\diamond}}, \quad|\alpha| \leq r+1
$$

A $2^{\prime}$.

$$
\int_{\mathbb{R}^{d}} x^{\alpha} \psi^{e}(x) d x=0, \quad|\alpha|<r
$$

Theorem 4.1. Let $M^{\diamond}>M>d$. Let the family of functions $\Phi_{n} \in C^{r+1}\left(\mathbb{R}^{d}\right)$, $n=1,2, \ldots$, satisfy $\boldsymbol{C 1 - 2}$ and let $\psi \in \Psi$ be any of Meyer's tensor product wavelets. Then for any $\varepsilon>0$ there exist $K \geq 1$ and $\theta \in \Theta_{K}$ such that

$$
\left|\psi^{(\alpha)}(x)-\theta^{(\alpha)}(x)\right| \leq \varepsilon(1+|x|)^{-M}, \quad|\alpha| \leq r,
$$

and

$$
\int_{\mathbb{R}^{d}} x^{\alpha} \theta(x) d x=0, \quad|\alpha|<r .
$$

Before proving Theorem 4.1 we give our main result:

Theorem 4.2. Let the parameters $s, p, q, r$, and $M$ be as in the assumptions of Theorem 3.1 or Theorem 3.2. Let $\Phi_{n} \in C^{r+1}\left(\mathbb{R}^{d}\right), n=1,2, \ldots$, satisfy C1-2 with $M^{\diamond}>M$. Then there exist $K \geq 1$ and a set of functions $\left\{\theta^{e}: e \in E\right\} \subset \Theta_{K}$ such that $B:=\left\{\theta_{I}^{e}: I \in D, e \in E\right\}$ is an unconditional basis for $\dot{F}_{p q}^{s}$ or $\dot{B}_{p q}^{s}$, respectively.

In particular, there exist bases for $\dot{F}_{p q}^{s}$ and $\dot{B}_{p q}^{s}, B_{R}:=\left\{R_{I}^{e}: I \in D, e \in E\right\}$ and $B_{G}:=\left\{G_{I}^{e}: I \in D, e \in E\right\}$, where $R^{e}$ is a rational function of the form

$$
R^{e}(\cdot)=\sum_{j=1}^{\mu} \frac{c_{j}}{\left(1+n^{2}\left|\cdot-a_{j}\right|^{2}\right)^{N}}, \quad n \mu \leq K\left(N>M^{\diamond} / 2\right),
$$

and $G^{e}$ is of the form

$$
G^{e}(\cdot)=\sum_{j=1}^{\mu} c_{j} e^{-n^{2}\left|\cdot-a_{j}\right|^{2}}, \quad n \mu \leq K
$$


Proof. The first part of Theorem 4.2 is immediate from Theorems 3.1$] 3.2$, and 4.1 For the proof of the second part, it suffices to note that $\Phi_{R, n}(\cdot):=C_{1} n^{d} e^{-n^{2}|\cdot|^{2}}$ and $\Phi_{G, n}(\cdot):=C_{2} n^{d}\left(1+n^{2}|\cdot|^{2}\right)^{-N}\left(N>M^{\diamond} / 2\right)$ satisfy $\mathbf{C 1 - 2}$ with $\mu=1$.

Proof of Theorem 4.1. The first step is to show that for every function $g$ satisfying A1' there exists $\tilde{\theta} \in \Theta_{K}$ such that

$$
\left|g^{(\alpha)}(x)-\tilde{\theta}^{(\alpha)}(x)\right| \leq \varepsilon(1+|x|)^{-M}, \quad|\alpha| \leq r .
$$

We first approximate $g$ by the convolution operator $\omega_{n}:=g * \Phi_{n}$. Taking into account C2, we have

$$
g^{(\alpha)}(x)-\omega_{n}^{(\alpha)}(x)=\int_{\mathbb{R}^{d}}\left[g^{(\alpha)}(x)-g^{(\alpha)}(x-y)\right] \Phi_{n}(y) d y, \quad|\alpha| \leq r .
$$

We denote $U:=n^{\eta / 2 M}$, where $\eta:=\min \left\{1, M^{\diamond}-M\right\}>0$. We will consider two cases for $x$.

CASE I: $|x| \leq U$. By $\mathbf{A} \mathbf{1}^{\prime}$, it follows that

$$
\left|g^{(\alpha)}(x-y)-g^{(\alpha)}(x)\right| \leq C \min \{1,|y|\} .
$$

From this and $\mathbf{C 1}$, we find, by simple calculation,

$$
\begin{aligned}
\left|g^{(\alpha)}(x)-\omega_{n}^{(\alpha)}(x)\right| & \leq C \int_{\mathbb{R}^{d}} \frac{\min \{1,|y|\} n^{d}}{(1+n|y|)^{M^{\diamond}}} d y \leq C\left(n^{-1}+n^{-M^{\diamond}+d}\right) \\
& \leq C n^{-\eta}=\frac{C n^{-\eta / 2}}{U^{M}} \leq \frac{C n^{-\eta / 2}}{(1+|x|)^{M}} .
\end{aligned}
$$

CASE II: $|x|>U$. We will integrate over $\Omega:=\{y:|y| \leq|x| / 2\}$ and $\Omega^{c}$. If $y \in \Omega$, then $|x-y| \geq|x| / 2$ and we find, by $\mathbf{A} \mathbf{1}^{\prime}$,

$$
\left|g^{(\alpha)}(x-y)-g^{(\alpha)}(x)\right| \leq\left|g^{(\alpha)}(x-y)\right|+\left|g^{(\alpha)}(x)\right| \leq \frac{C}{(1+|x|)^{M^{\diamond}}} .
$$

Therefore,

$$
\begin{aligned}
& \int_{\Omega}\left|g^{(\alpha)}(x-y)-g^{(\alpha)}(x)\right|\left|\Phi_{n}(y)\right| d y \leq \frac{C}{(1+|x|)^{M^{\diamond}}} \int_{\mathbb{R}^{d}}\left|\Phi_{n}(y)\right| d y \\
& \leq \frac{C}{(1+|x|)^{M^{\diamond}}} \leq \frac{C}{(1+|U|)^{\eta}(1+|x|)^{M}} \leq \frac{C n^{-\eta^{2} / 2 M}}{(1+|x|)^{M}}
\end{aligned}
$$

We now integrate over $\Omega^{c}:=\{y:|y|>|x| / 2\}$. We use $\mathbf{A} \mathbf{1}^{\prime}$, C1, and that $|x|>U$ to find

$$
\begin{aligned}
& \int_{\Omega^{c}}\left|g^{(\alpha)}(x-y)-g^{(\alpha)}(x)\right|\left|\Phi_{n}(y)\right| d y \\
& \leq \int_{\Omega^{c}}\left|g^{(\alpha)}(x-y)\right|\left|\Phi_{n}(y)\right| d y+\int_{\Omega^{c}}\left|g^{(\alpha)}(x)\right|\left|\Phi_{n}(y)\right| d y \\
& \leq \int_{\Omega^{c}} \frac{C n^{d}}{(1+|x-y|)^{M^{\diamond}}(1+n|y|)^{M^{\diamond}}} d y+\frac{C}{(1+|x|)^{M^{\diamond}}} \int_{\mathbb{R}^{d}}\left|\Phi_{n}(y)\right| d y \\
& \leq \frac{C n^{d}}{(1+n|x|)^{M^{\diamond}}} \int_{\mathbb{R}^{d}} \frac{1}{(1+|x-y|)^{M^{\diamond}}} d y+\frac{C}{(1+|x|)^{M^{\diamond}}} \\
& \leq \frac{C}{(1+|x|)^{M^{\diamond}}} \leq \frac{C n^{-\eta^{2} / 2 M}}{(1+|x|)^{M}} .
\end{aligned}
$$


Putting together the above estimates, we obtain, for sufficiently large $n$,

$$
\left|g^{(\alpha)}(x)-\omega_{n}^{(\alpha)}(x)\right| \leq \frac{C n^{-\eta^{2} / 2 M}}{(1+|x|)^{M}} \leq \frac{\varepsilon}{(1+|x|)^{M}}
$$

Now, we fix $n$ and truncate the integral for $\omega_{n}$. We denote $Q:=\left[-2^{q}, 2^{q}\right]^{d}$, where $q \in \mathbb{Z}_{+}$will be specified later on. We define

$$
\lambda_{q}(x):=\int_{Q} g(y) \Phi_{n}(x-y) d y
$$

Evidently, we have

$$
\omega_{n}^{(\alpha)}(x)-\lambda_{q}^{(\alpha)}(x)=\int_{\mathbb{R}^{d} \backslash Q} g(y) \Phi_{n}^{(\alpha)}(x-y) d y, \quad|\alpha| \leq r .
$$

Using $\mathbf{A} \mathbf{1}^{\prime}$ and $\mathbf{C 1}$, we get

$$
\left|\omega_{n}^{(\alpha)}(x)-\lambda_{q}^{(\alpha)}(x)\right| \leq C \int_{\mathbb{R}^{d} \backslash Q} \frac{n^{r \mu+d}}{(1+|y|)^{M^{\diamond}}(1+n|x-y|)^{M^{\diamond}}} d y=: L .
$$

To estimate the latter integral we consider different cases for $x$.

CASE I: $|x| \leq 2^{q} / 2$. For $y \in \mathbb{R}^{d} \backslash Q$, we have $|x-y| \geq 2^{q} / 2 \geq|x|$ and $|y| \geq 2^{q} \geq 2|x|$ and hence

$$
\begin{aligned}
L & \leq \frac{C n^{r \mu}}{(1+|x|)^{M^{\diamond}}} \int_{\mathbb{R}^{d} \backslash Q} \frac{n^{d}}{(1+n|x-y|)^{M^{\diamond}}} d y \\
& \leq \frac{C n^{r \mu}}{(1+|x|)^{M^{\diamond}}} \int_{|u| \geq 2^{q-1}} \frac{n^{d}}{(1+n|u|)^{M^{\diamond}}} d u \leq \frac{C n^{r \mu-M^{\diamond}+d} 2^{-M^{\diamond}+d}}{(1+|x|)^{M^{\diamond}}} .
\end{aligned}
$$

CASE II: $|x|>2^{q} / 2$. We will integrate over $\Omega:=\left(\mathbb{R}^{d} \backslash Q\right) \cap\{y:|y| \leq|x| / 2\}(\Omega$ can be empty) and over $\Omega^{\prime}:=\left(\mathbb{R}^{d} \backslash Q\right) \backslash \Omega$. If $y \in \Omega$, then $|x-y| \geq|x| / 2$ and hence

$$
\begin{aligned}
\int_{\Omega} \frac{n^{r \mu+d}}{(1+|y|)^{M^{\diamond}}(1+n|x-y|)^{M^{\diamond}}} d y & \leq \frac{C n^{r \mu+d}}{(1+n|x|)^{M^{\diamond}}} \int_{\mathbb{R}^{d}} \frac{1}{(1+|y|)^{M^{\diamond}}} d y \\
& \leq \frac{C n^{r \mu-M^{\diamond}+d}}{(1+|x|)^{M^{\diamond}}} \leq \frac{C n^{r \mu} 2^{-q \eta}}{(1+|x|)^{M}} .
\end{aligned}
$$

If $y \in \Omega^{\prime}$, then $|y|>|x| / 2$ and hence

$$
\begin{aligned}
\int_{\Omega^{\prime}} \frac{n^{r \mu+d}}{(1+|y|)^{M^{\diamond}}(1+n|x-y|)^{M^{\diamond}}} d y & \leq \frac{C n^{r \mu}}{(1+|x|)^{M^{\diamond}}} \int_{\mathbb{R}^{d}} \frac{n^{d}}{(1+n|x-y|)^{M^{\diamond}}} d y \\
& \leq \frac{C n^{r \mu-M^{\diamond}+d}}{(1+|x|)^{M^{\diamond}}} \leq \frac{C n^{r \mu} 2^{-q \eta}}{(1+|x|)^{M}} .
\end{aligned}
$$

Therefore, we have in both cases

$$
L \leq \frac{C n^{r \mu} 2^{-q \eta}}{(1+|x|)^{M}}
$$

Selecting $q \in \mathbb{Z}_{+}$sufficiently large, we obtain

$$
\left|\omega_{n}^{(\alpha)}(x)-\lambda_{q}^{(\alpha)}(x)\right| \leq \frac{\varepsilon}{(1+|x|)^{M}}, \quad|\alpha| \leq r .
$$

We fix $q$. 
Next, we will discretize the integral for $\lambda_{q}$, in order for our approximant to be in $\Theta_{K}$ for some $K \geq 1$. We let $\mathcal{Q}_{m}$ denote the set of all dyadic subcubes of $Q$ of side-length $2^{-m}$, where $m \in \mathbb{Z}_{+}$will be selected later on. We define

$$
\theta_{m}(\cdot):=\sum_{I \in \mathcal{Q}_{m}}|I| g\left(x_{I}\right) \Phi_{n}\left(\cdot-x_{I}\right) .
$$

Note that $\theta_{m} \in \Theta_{n 2^{d(q+m+1)}}$.

We have, for $|\alpha| \leq r$,

$$
\begin{aligned}
\lambda_{q}^{(\alpha)}(x)-\theta_{m}^{(\alpha)}(x) & =\sum_{I \in \mathcal{Q}_{m}} \int_{I}\left[g(y) \Phi_{n}^{(\alpha)}(x-y)-g\left(x_{I}\right) \Phi_{n}^{(\alpha)}\left(x-x_{I}\right)\right] d y \\
& =\sum_{I \in \mathcal{Q}_{m}} \int_{I}\left[F(y)-F\left(x_{I}\right)\right] d y
\end{aligned}
$$

where $F(\cdot):=g(\cdot) \Phi_{n}^{(\alpha)}(x-\cdot)$. It follows that

$$
\begin{aligned}
\left|\theta_{m}^{(\alpha)}(x)-\lambda_{n}^{(\alpha)}(x)\right| & =\left|\sum_{I \in \mathcal{Q}_{m}} \int_{I} \sum_{|\eta|=1} \int_{0}^{1}\left(y-x_{I}\right)^{\eta} F^{(\eta)}\left(x_{I}+t\left(y-x_{I}\right)\right) d t d y\right| \\
& \leq C \sum_{I \in \mathcal{Q}_{m}} \ell(I) \int_{I} \max _{\substack{z \in\left[x_{I}, y\right] \\
|\eta|=1}}\left|F^{(\eta)}(z)\right| d y \\
& \leq C 2^{-m} \max _{\substack{z \in Q \\
|\eta|=1}}\left|F^{(\eta)}(z)\right|,
\end{aligned}
$$

where $\left[x_{I}, y\right]$ is the line-segment joining $x_{I}$ with $y$. By the product rule, we have

$$
F^{(\eta)}(z)=\sum_{\gamma \leq \eta} c_{\gamma} g^{(\eta-\gamma)}(z) \Phi_{n}^{(\alpha+\gamma)}(x-z) .
$$

If $|x| \leq 2 \sqrt{d} 2^{q}$ and $z \in Q$, then

$$
\left|F^{(\eta)}(z)\right| \leq C \sum_{\gamma \leq \eta}\left|\Phi_{n}^{(\alpha+\gamma)}(x-z)\right| \leq C n^{(r+1) \mu+d} \leq \frac{C n^{(r+1) \mu+d} 2^{q M}}{(1+|x|)^{M}} .
$$

If $|x|>2 \sqrt{d} 2^{q}$ and $z \in Q$, then $|x-z| \geq|x| / 2$ and hence

$$
\left|F^{(\eta)}(z)\right| \leq C \sum_{\gamma \leq \eta}\left|\Phi_{n}^{(\alpha+\gamma)}(x-z)\right| \leq \frac{C n^{(r+1) \mu+d}}{(1+|n x|)^{M}} \leq \frac{C n^{(r+1) \mu+d-M}}{(1+|x|)^{M}} .
$$

In both cases by selecting $m$ large enough ( $n$ and $q$ are fixed), we obtain

$$
\left|\theta_{m}^{(\alpha)}(x)-\lambda_{q}^{(\alpha)}(x)\right| \leq \frac{\varepsilon}{(1+|x|)^{M}} .
$$

From (4.4) - (4.6), we conclude that for any $\varepsilon>0$ there exist $K \geq 1$ and $\tilde{\theta} \in \Theta_{K}$ $\left(K:=n 2^{d(q+m+1)}, \tilde{\theta}:=\theta_{m}\right)$ such that

$$
\left|g^{(\alpha)}(x)-\tilde{\theta}^{(\alpha)}(x)\right| \leq \frac{3 \varepsilon}{(1+|x|)^{M}}, \quad|\alpha| \leq r .
$$

The second step is to show that, using the result of the first step, there exists $\theta \in \Theta_{K}$ which satisfies both (4.1) and (4.2). To make it easier, we shall use some of the specific properties of Meyer's wavelets. As usual, we denote by $\widehat{f}$ the Fourier transform of $f\left(\widehat{f}(\xi):=\int f(x) e^{-i x \xi} d x\right)$. We let $\psi$ denote Meyer's univariate wavelet 
and let $\phi$ be the associated scaling function (see [M] ). We recall that $\widehat{\psi}, \widehat{\phi} \in C^{\infty}, \widehat{\psi}$ is supported on $[-8 \pi / 3,-2 \pi / 3] \cup[2 \pi / 3,8 \pi / 3]$ and $\widehat{\phi}$ is supported on $[-4 \pi / 3,4 \pi / 3]$.

Let now $e$ be a nonzero vertex of the unit cube $E$ in $\mathbb{R}^{d}$ and let $\psi^{e}$ be the corresponding tensor product wavelet (see $\S 1.1$ ), i.e.,

$$
\psi^{e}(x):=\psi^{e_{1}}\left(x_{1}\right) \ldots \psi^{e_{d}}\left(x_{d}\right), \quad x \in \mathbb{R}^{d},
$$

where

It follows that

$$
\psi^{e_{i}}= \begin{cases}\phi, & e_{i}=0 \\ \psi, & e_{i}=1\end{cases}
$$

$$
\widehat{\psi^{e}}(\xi)=\widehat{\psi^{e_{1}}}\left(\xi_{1}\right) \ldots \widehat{\psi^{e_{d}}}\left(\xi_{d}\right)
$$

where at least one of the components of $e$ is 1 . Suppose $e_{j}=1$ for some $1 \leq j \leq d$. We let $v_{j}$ denote the unit vector in the direction of the $x_{j}$-axis. We shall next utilize the following two simple facts:

(a) $\widehat{\Delta_{h v_{j}}^{r} f}(\xi)=\left(e^{i h \xi_{j}}-1\right)^{r} \widehat{f}(\xi)$, where

$$
\left(\Delta_{h v_{j}}^{r} f\right)(x):=\sum_{k=0}^{r}(-1)^{r+k}\left(\begin{array}{l}
r \\
k
\end{array}\right) f\left(x+k h v_{j}\right)
$$

is the $r$-th difference of $f$ with step $h \in \mathbb{R}$ in the direction of $v_{j}$.

(b) If $|f(x)| \leq C(1+|x|)^{-M^{\diamond}}, M^{\diamond}>d+r$, then

$$
\int_{\mathbb{R}^{d}} x^{\alpha}\left(\Delta_{h v_{j}}^{r} f\right)(x) d x=\int_{\mathbb{R}^{d}}\left(\Delta_{-h v_{j}}^{r} x^{\alpha}\right)(x) f(x) d x=0, \quad|\alpha|<r .
$$

We define a function $g$ by the identity

$$
\widehat{g}(\xi):=\frac{\widehat{\psi^{e}}(\xi)}{\left(e^{i \xi_{j} / 2}-1\right)^{r}}=\widehat{\psi^{e_{1}}}\left(\xi_{1}\right) \cdots \frac{\widehat{\psi}\left(\xi_{j}\right)}{\left(e^{i \xi_{j} / 2}-1\right)^{r}} \cdots \widehat{\psi^{e_{d}}}\left(\xi_{d}\right) .
$$

Clearly, $\left(e^{i w / 2}-1\right)^{r}$ vanishes only at the integer multiples of $4 \pi$ which are not in the support of $\widehat{\psi}$ and hence $\frac{\widehat{\psi}(w)}{\left(e^{i w / 2}-1\right)^{r}} \in \mathcal{S}(\mathbb{R})$. Therefore, $\widehat{g} \in \mathcal{S}$ and hence $g \in \mathcal{S}$. By the above definition, $\widehat{\psi^{e}}(\xi)=\left(e^{i \xi_{j} / 2}-1\right)^{r} \widehat{g}(\xi)$ and hence $\psi^{e}=\Delta_{v_{j} / 2}^{r} g$.

Now, using the result from the first step of the proof (see (4.7)), there exists $\tilde{\theta} \in \Theta_{K}$ such that

$$
\left|g^{(\alpha)}(x)-\tilde{\theta}^{(\alpha)}(x)\right| \leq \frac{3 \varepsilon}{(1+|x|)^{M}}, \quad|\alpha| \leq r,
$$

and

$$
|\tilde{\theta}(x)| \leq \frac{C}{(1+|x|)^{M^{\diamond}}}, \quad M^{\diamond}>d+r .
$$

We define $\theta:=\Delta_{v_{j} / 2}^{r} \tilde{\theta}$. Evidently, $\theta \in \Theta_{(r+1) K}$. We have $\psi^{e}-\theta=\Delta_{v_{j} / 2}^{r}(g-\tilde{\theta})$ and, using (4.8),

$$
\left|\left(\psi^{e}\right)^{(\alpha)}(x)-\theta^{(\alpha)}(x)\right| \leq C \frac{\varepsilon}{(1+|x|)^{M}}, \quad|\alpha| \leq r,
$$

where $C$ is independent of $\varepsilon$. On the other hand, from $\theta:=\Delta_{v_{j} / 2}^{r} \tilde{\theta}$ and [4.9), it follows that

$$
\int_{\mathbb{R}^{d}} x^{\alpha} \theta(x) d x=0, \quad|\alpha|<r .
$$

This completes the proof of the theorem. 
- Construction of bases from shifts and dilates of a single function satisfying the Strang-Fix conditions.

Let $\Phi$ be a function in $C^{r}\left(\mathbb{R}^{d}\right)(r \geq 1)$ which satisfies the following conditions:

D1. There exists $M^{\diamond}>d+r+1$ such that

$$
\left|\Phi^{(\alpha)}(x)\right| \leq C(1+|x|)^{-M^{\diamond}}, \quad 0 \leq|\alpha| \leq r .
$$

D2. The shifts of $\Phi$ reproduce the polynomials of degree $\leq r$, i.e.,

$$
\sum_{j \in \mathbb{Z}^{d}} j^{\alpha} \Phi(x-j)=x^{\alpha}, \quad|\alpha| \leq r .
$$

We note that functions that satisfy D1-2 play an important role in the study of the approximation properties of shift-invariant spaces generated by piecewise polynomial functions or radial basis functions (see $[\mathrm{BR}]$ ). Moreover, the original construction of wavelets is based upon a function $\Phi$ that satisfies D1-2. However, additional assumptions on $\Phi$ are required such as the stability of the shifts of $\Phi$ and a refinement equation $\Phi(\cdot)=\sum_{j \in \mathbb{Z}^{d}} a_{j} \Phi(2 \cdot-j)$. Our goal in this part is to construct a basis for the Triebel-Lizorkin and Besov spaces from the shifts and dilates of a function $\Phi$ without any recourse to either the refinement equation or the stability of its shifts. Moreover, the present construction will be considerably simpler than the one from Theorem 4.1

Let $0<h \leq 1$. Similarly as in Theorem 4.1, we define

$$
\Theta_{h}:=\left\{\theta: \theta(\cdot)=\sum_{j \in L} a_{j} \Phi(\cdot / h-j), \# L \leq C h^{-d}\right\},
$$

where $L \subset \mathbb{Z}^{d}, \# L$ is the number of its elements, and the constant $C$ is independent of $h$.

In what follows, we assume that $\psi\left(\psi\right.$ being any $\left.\psi^{e} \in \Psi\right)$ is a compactly supported function in $C^{\rho}\left(\mathbb{R}^{d}\right)$, with $\rho>\max \{r, d\}$ which satisfies:

A2'.

$$
\int_{\mathbb{R}^{d}} x^{\alpha} \psi(x) d x=0, \quad|\alpha|<r .
$$

Theorem 4.3. Let $M^{\diamond}>M+r+1, M>d$, and $\Phi \in C^{r}\left(\mathbb{R}^{d}\right)$ satisfy D1-2. Let also $\psi$ be a compactly supported function in $C^{\rho}\left(\mathbb{R}^{d}\right)$ satisfying $\mathbf{A} \mathbf{2}^{\prime}$. Then for any $\varepsilon>0$ there exist $0<h \leq 1$ and $\theta \in \Theta_{h}$ such that

$$
\left|\psi^{(\alpha)}(x)-\theta^{(\alpha)}(x)\right| \leq \varepsilon(1+|x|)^{-M}, \quad|\alpha| \leq r
$$

and

$$
\int_{\mathbb{R}^{d}} x^{\alpha} \theta(x) d x=0, \quad|\alpha|<r .
$$

As a consequence of Theorem 4.3 we immediately get

Theorem 4.4. Let the parameters $s, p, q, r$, and $M$ be as in the assumptions of Theorem 3.1 or Theorem 3.2 Let $\Phi \in C^{r}\left(\mathbb{R}^{d}\right)$, satisfy D1-2 with $M^{\diamond}>M+r+1$. Then there exist $h>0$ and a set of functions $\left\{\theta^{e}: e \in E\right\} \subset \Theta_{h}$ such that $B:=\left\{\theta_{I}^{e}: I \in D, e \in E\right\}$ is an unconditional basis for $\dot{F}_{p q}^{s}$ or $\dot{B}_{p q}^{s}$, respectively.

In the proof of Theorem 4.3 we will use the well known fact (see, e.g, [K2]) that if $\widehat{\Phi}(0)=1$ and

$$
\Phi(\cdot) \leq C(1+|\cdot|)^{-M^{\diamond}}, \quad M^{\diamond}>d+r+1
$$


then D2 is equivalent to

$$
\widehat{\Phi}(0)=1, \quad \widehat{\Phi}^{(\alpha)}(0)=0, \quad 1 \leq|\alpha| \leq r,
$$

and

$$
\widehat{\Phi}^{(\alpha)}(2 j \pi)=0, \quad j \in \mathbb{Z}^{d} \backslash\{0\}, \quad|\alpha| \leq r .
$$

Conditions (4.13) are called the Strang-Fix conditions.

Proof of Theorem 4.3. Let $\gamma$ be any compactly supported function in $C^{\rho}\left(\mathbb{R}^{d}\right)$ satisfying (4.12) and (4.13). Without loss of generality we will assume that both $\psi$ and $\gamma$ are supported on the unit cube $[0,1]^{d}$. We define

$$
P_{h} \psi(x):=\sum_{j \in \mathbb{Z}^{d}} c_{h}(j) \Phi(x / h-j) \quad \text { with } \quad c_{h}(j):=h^{-d} \int_{\mathbb{R}^{d}} \psi(y) \overline{\gamma(y / h-k)} d y .
$$

Since $\gamma$ and $\psi$ are supported on $[0,1]^{d}$, the above sum involves $\leq C h^{-d}$ terms. To simplify our notation we write

$$
P_{h} \psi(x)=h^{-d} \int_{\mathbb{R}^{d}} \psi(y) R(x / h, y / h) d y, \quad R(x, y):=\sum_{j \in \mathbb{Z}^{d}} \overline{\gamma(y-j)} \Phi(x-j) .
$$

Our goal is to prove that, for $h>0$ sufficiently small, $P_{h} \psi$ satisfies all the desired properties of $\theta$. Then we will define $\theta:=P_{h} \psi$. We start by proving that if $h>0$ is small enough then

$$
\left|\psi^{(\alpha)}(x)-\left(P_{h} \psi\right)^{(\alpha)}(x)\right| \leq \varepsilon(1+|x|)^{-M}, \quad|\alpha| \leq r .
$$

The assumptions on $\Phi$ guarantee that $h^{-d} \int R(x / h, y / h) d y=1$ for every $x \in \mathbb{R}^{d}$ (see $[\mathrm{K} 2]$ ), and that (see $[\mathrm{M}]$ )

$$
\psi(x)=\lim _{h \rightarrow 0} P_{h} \psi(x) \quad \text { for almost all } x .
$$

It follows that

$$
\begin{aligned}
\psi(x)-P_{h} \psi(x) & =\sum_{i=0}^{\infty}\left[P_{h 2^{-i}} \psi(x)-P_{h 2^{-i-1}} \psi(x)\right] \\
& =\sum_{i=0}^{\infty} T_{h 2^{-i}} \psi(x)
\end{aligned}
$$

where

$$
T_{h} \psi(x):=h^{-d} \int_{\mathbb{R}^{d}} \psi(y) K(x / h, y / h) d y \quad \text { with } \quad K(x, y):=R(x, y)-2^{d} R(2 x, 2 y) .
$$

By the assumptions on $\Phi$, it is easily seen that

$$
\left|K_{x}^{(\alpha)}(x, y)\right| \leq C(1+|x-y|)^{-M^{\diamond}}, \quad 0 \leq|\alpha| \leq r,
$$

where $K_{x}^{(\alpha)}$ is the partial derivative in $x$. Moreover,

$$
P_{h}\left(x^{\alpha}\right)=x^{\alpha}, \quad 0 \leq|\alpha| \leq r, h>0,
$$

which implies that $T_{h}\left(x^{\alpha}\right)=0,0 \leq|\alpha| \leq r$ (see [K2]).

We claim that for every $0 \leq|\alpha| \leq r$ and $0<h \leq 1$

$$
\left|\left(T_{h} \psi\right)^{(\alpha)}(x)\right| \leq C h^{r+1-|\alpha|}(1+|x|)^{-M},
$$

where the constant is independent of $h$.

We consider two cases for $x$ : 
CASE I: $|x|>2 \sqrt{d}$. Then for every $y \in[0,1]^{d}$ we have $|y| \leq|x| / 2$ and therefore $(1+|x-y| / h) \geq C(1+|x|) / h$. Since $\psi$ is supported on the unit cube it follows that

$$
\begin{aligned}
& \left|\left(T_{h} \psi\right)^{(\alpha)}(x)\right| \leq h^{-d-|\alpha|} \int_{[0,1]^{d}}\left|K_{x}^{(\alpha)}(x / h, y / h)\right||\psi(y)| d y \\
& \leq C h^{-d-|\alpha|} \int_{[0,1]^{d}} \frac{1}{(1+|x / h-y / h|)^{M^{\diamond}}} \mid(\psi(y) \mid d y \\
& \leq C h^{M^{\diamond}-d-|\alpha|}(1+|x|)^{-M^{\diamond}} \\
& \leq C h^{r+1-|\alpha|}(1+|x|)^{-M}
\end{aligned}
$$

because $M^{\diamond}>d+r+1$.

CASE II: $|x| \leq 2 \sqrt{d}$. Then

$$
\begin{aligned}
& \left|\left(T_{h} \psi\right)^{(\alpha)}(x)\right|=h^{-d-|\alpha|}\left|\int_{\mathbb{R}^{d}} K_{x}^{(\alpha)}(x / h, y / h) \psi(y) d y\right| \\
& =h^{-d-|\alpha|}\left|\int_{\mathbb{R}^{d}} K_{x}^{(\alpha)}(x / h, y / h)\left(\psi(y)-\sum_{0 \leq|\eta|<r+1} \frac{\psi^{(\eta)}(x)}{\eta !}(y-x)^{\eta}\right) d y\right| \\
& =h^{-d-|\alpha|}\left|\int_{\mathbb{R}^{d}} K_{x}^{(\alpha)}(x / h, y / h) \sum_{|\eta|=r+1} \int_{0}^{1} \frac{(y-x)^{\eta}}{\eta !} \psi^{(\eta)}(x+t(y-x)) t^{r} d t d y\right| \\
& \leq C h^{-d-|\alpha|+r+1} \int_{\mathbb{R}^{d}} \frac{1}{(1+|x / h-y / h|)^{M^{\diamond}-r-1}} \sup _{\substack{z \in[x, y] \\
|\eta|=r+1}}\left|(\psi)^{(\eta)}(z)\right| d y \\
& \leq C h^{r+1-|\alpha|} .
\end{aligned}
$$

Therefore, for $0<h \leq 1$,

$$
\begin{aligned}
\left|\psi^{(\alpha)}(x)-\left(P_{h} \psi\right)^{(\alpha)}(x)\right| & \leq \sum_{i=0}^{\infty}\left|T_{h 2^{-i}}^{(\alpha)} \psi(x)\right| \leq C \sum_{i=0}^{\infty}\left(\frac{h}{2^{i}}\right)^{r+1-|\alpha|}(1+|x|)^{-M} \\
& \leq C h(1+|x|)^{-M} .
\end{aligned}
$$

Thus by choosing $0<h<\varepsilon / C$ (4.14) holds.

Next, we will prove the moment conditions. We have

$$
\int_{\mathbb{R}^{d}} x^{\alpha} \psi(x) d x=(-i)^{|\alpha|} \widehat{\psi}^{(\alpha)}(0)=0, \quad|\alpha|<r .
$$

We also note that

$$
\int x^{\alpha} P_{h} \psi(x)=0 \Longleftrightarrow{\widehat{P_{h} \psi}}^{(\alpha)}(0)=0, \quad 0 \leq|\alpha|<r, h>0,
$$

However, since (see [JM, Theorem 3.2])

$$
\widehat{P_{h} \psi}(\xi)=\sum_{j \in \mathbb{Z}} \widehat{\psi}(\xi+2 j \pi / h) \bar{\gamma}(h \xi+2 j \pi) \widehat{\Phi}(h \xi)
$$

for $\rho>d$ the decay of $\widehat{\psi}$ and $\widehat{\gamma}$ allows to differentiate term by term and using the Strang-Fix conditions (4.13), (4.16), and Leibnitz's formula the result follows. 


\section{Application OF THE NEW BASES TO NONLINEAR APPROXIMATION}

In this section, we consider nonlinear $n$-term approximation by shifts and dilates of a single function $\Phi$. We define

$$
\begin{gathered}
\mathcal{D}:=\mathcal{D}(\Phi):=\left\{\Phi(a \cdot+b): a \in \mathbb{R}, b \in \mathbb{R}^{d}\right\}, \\
\mathcal{D}_{n}:=\mathcal{D}_{n}(\Phi):=\left\{S: S=\sum_{j=1}^{n} a_{j} \Phi_{j}, \Phi_{j} \in \mathcal{D}\right\} .
\end{gathered}
$$

We now consider the best $n$-term approximation of $f$ from $\mathcal{D}$ in the norm $\|\cdot\|_{p}$ in $L_{p}$ if $1<p<\infty$ or $H_{p}$ if $0<p \leq 1$, defined by

$$
\sigma_{n}(f, \mathcal{D})_{p}:=\inf _{S \in \mathcal{D}_{n}}\|f-S\|_{p}
$$

We are particularly interested in the $n$-term approximation from $\mathcal{D}(\Phi)$, where $\Phi$ is the rational function $\Phi(\cdot):=R(\cdot):=\left(1+|\cdot|^{2}\right)^{-N}$ with $N$ big enough but fixed, or $\Phi(\cdot):=G(\cdot):=e^{-|\cdot|^{2}}$. Thus $\mathcal{D}_{n}(R)$ is the set of all rational functions $R_{n}$ on $\mathbb{R}^{d}$ of the form

$$
R_{n}(\cdot)=\sum_{j=1}^{n} \frac{c_{j}}{\left(1+\left|a_{j} \cdot+b_{j}\right|^{2}\right)^{N}}
$$

and $\mathcal{D}_{n}(G)$ is the set of all functions $G_{n}$ of the form

$$
G_{n}(\cdot)=\sum_{j=1}^{n} c_{j} e^{-\left|a_{j} \cdot+b_{j}\right|^{2}} .
$$

We denote by $R_{n}(f)_{p}$ and $G_{n}(f)_{p}$ the best approximation of $f \in H_{p}(0<p<\infty)$ from $\mathcal{D}_{n}(R)$ and $\mathcal{D}_{n}(G)$, respectively. that

We let $A_{p q}^{\gamma}:=A_{p q}^{\gamma}(\mathcal{D})$ denote the approximation space of all functions $f$ such

$$
|f|_{A_{p q}^{\gamma}}:=\left(\sum_{n=1}^{\infty}\left(n^{\gamma} \sigma_{n}(f, \mathcal{D})_{p}\right)^{q} \frac{1}{n}\right)^{1 / q}<\infty
$$

with the $\ell_{q}$-norm replaced by the sup-norm if $q=\infty$ as usual.

A basic problem is to characterize the approximation spaces $A_{p q}^{\gamma}$ for a given function $\Phi$. We refer the reader to [De as a general reference for nonlinear approximation.

We now assume that the family $\left\{\Phi_{n}: n=1,2, \ldots\right\} \subset \mathcal{D}$ satisfies C1-2 $(\S 4)$ and Theorem 4.2 applies with the parameters selected so that the resulting basis $B=\left\{\theta_{I}^{e}: I \in D, e \in E\right\}$ is an unconditional basis for $\dot{F}_{p 2}^{0}, 0<p<\infty$, and $\dot{B}_{\tau \tau}^{s}$, $s>0$ and $1 / \tau:=s / d+1 / p$. Note that $\dot{F}_{p 2}^{0} \approx H_{p} \approx L_{p}$ if $1<p<\infty$ and $\dot{F}_{p 2}^{0} \approx H_{p}$ if $0<p \leq 1$.

We also remark that there exist bases $B=\left\{\theta_{I}^{e}: I \in D, e \in E\right\}$ like this with $\theta^{e} \in \mathcal{D}_{K}(R)$ or $\theta^{e} \in \mathcal{D}_{K}(G)$ (see Theorem 4.2).

We first consider $n$-term approximation from a basis $B$.

Theorem 5.1. Let $B$ be one of the above bases for $H_{p}, 0<p<\infty$, and $B_{\tau, \tau}^{s}$, $s>0,1 / \tau:=s / d+1 / p$. Then the following inequalities hold:

(Jackson inequality) $\quad \sigma_{n}(f, B)_{p} \leq C n^{-s / d}\|f\|_{\dot{B}_{\tau \tau}^{s}}, \quad f \in \dot{B}_{\tau \tau}^{s}$,

(Bernstein inequality) $\|S\|_{\dot{B}_{\tau \tau}^{s}} \leq C n^{s / d}\|S\|_{p}, \quad S \in B_{n}$,

where $B_{n}:=\left\{S: S=\sum_{j=1}^{n} a_{j} t_{j}, t_{j} \in B\right\}$. 
Proof. This theorem follows by Theorem 4.2 The proof can be carried out similarly as the proofs of Theorem 5, Corollary 1, and Theorem 6 from [De. We leave the details to the reader.

From Theorem 5.1 and standard arguments (see, e.g, DL or $[\mathrm{PP}]$ ), we obtain the following characterization of the approximation spaces $A_{p q}^{\gamma}(B)$ :

Theorem 5.2. We have, for $0<\alpha<s, 0<p<\infty$, and $0<q \leq \infty$,

$$
A_{p q}^{\alpha / d}(B)=\left(H_{p}, \dot{B}_{\tau \tau}^{s}\right)_{\alpha / s, q}
$$

with equivalent norms, where $(X, Y)_{\theta, q}$ is the real interpolation space between $X$ and $Y$. In particular, $A_{p q}^{\alpha / d}(B)=\dot{B}_{q q}^{\alpha}$ when $1 / q=1 / p+\alpha / d$.

We now return to the original problem for $n$-term approximation from $\mathcal{D}$. Since $\theta^{e} \in \mathcal{D}_{K}(K$ fixed $)$ for $e \in E$, Theorem 5.1 yields the following

Corollary 5.1. If $f \in \dot{B}_{\tau \tau}^{s}, s>0,1 / \tau:=s / d+1 / p, 0<p<\infty$, then

$$
\sigma_{n}(f, \mathcal{D})_{p} \leq C n^{-s / d}\|f\|_{\dot{B}_{\tau \tau}^{s}}
$$

and, in particular,

$$
R_{n}(f)_{p} \leq C n^{-s / d}\|f\|_{\dot{B}_{\tau \tau}^{s}}, \quad G_{n}(f)_{p} \leq C n^{-s / d}\|f\|_{\dot{B}_{\tau \tau}^{s}} .
$$

A natural problem arises: Whether for a given function $\Phi$ the following Bernstein type inequality holds:

$$
\|S\|_{\dot{B}_{\tau \tau}^{s}} \leq C n^{s / d}\|S\|_{p}, \quad S \in \mathcal{D}_{n}(\Phi)
$$

with $p, s, d$, and $\tau$ as above.

Clearly, if (5.1) holds for some function $\Phi$, then the corresponding approximation spaces $A_{p q}^{\gamma}(\mathcal{D})$ can be characterized by interpolation spaces as in Theorem 5.2 .

Note that, if $d=1$ and $\Phi(x)=\left(1+x^{2}\right)^{-N}$, the needed characterization of $A_{p q}^{\gamma}(\mathcal{D})(1<p<\infty)$ follows by Theorem 5.1 and the inverse estimate of Pekarskii (see [Pek] and the references therein).

\section{APPENDIX}

- Wavelet characterizations of Triebel-Lizorkin and Besov spaces. We begin with the definition of these spaces (see [Pee, [T]). Let $\phi \in \mathcal{S}$ be such that $\operatorname{supp} \widehat{\phi} \subset\left\{\xi: 2^{-1} \leq|\xi| \leq 2\right\}$ and $|\widehat{\phi}(\xi)| \geq c>0$ if $3 / 5 \leq|\xi| \leq 5 / 3$. We denote $\phi_{\nu}(\cdot):=2^{\nu d} \phi\left(2^{\nu} \cdot\right)$.

For $s \in \mathbb{R}, 0<p<\infty, 0<q \leq \infty$, the homogeneous Triebel-Lizorkin space $\dot{F}_{p q}^{s}$ is defined to be the set of all $f \in \mathcal{S}^{\prime} / \mathcal{P}$ such that

$$
\|f\|_{\dot{F}_{p q}^{s}}:= \begin{cases}\left\|\left(\sum_{\nu \in \mathbb{Z}}\left[2^{\nu s}\left|\phi_{\nu} * f\right|\right]^{q}\right)^{1 / q}\right\|_{L_{p}}, & q \neq \infty \\ \left\|\left(\sup _{\nu \in \mathbb{Z}} 2^{\nu s}\left|\phi_{\nu} * f\right|\right)\right\|_{L_{p}}, & q=\infty\end{cases}
$$

is finite.

In a similar vein, for $s \in \mathbb{R}, 0<p, q \leq \infty$, the homogeneous Besov space $\dot{B}_{p q}^{s}$ is defined to be the set of all $f \in \mathcal{S}^{\prime} / \mathcal{P}$ such that

$$
\|f\|_{\dot{B}_{p q}^{s}}:=\left\{\begin{array}{cl}
\left(\sum_{\nu \in \mathbb{Z}}\left[2^{\nu s}\left\|\phi_{\nu} * f\right\|_{L_{p}}\right]^{q}\right)^{1 / q}, & q \neq \infty \\
\sup _{\nu \in \mathbb{Z}} 2^{\nu s}\left\|\phi_{\nu} * f\right\|_{L_{p}}, & q=\infty
\end{array}\right.
$$

is finite. 
Let now $\Psi:=\left\{\psi^{e}: e \in E\right\}$ be a set of orthogonal wavelets for $L_{2}\left(\mathbb{R}^{d}\right)$ which satisfy the following two conditions:

A1. $\Psi \subset C^{r}$ and

$$
\left|\left(\psi^{e}\right)^{(\alpha)}(x)\right| \leq C(1+|x|)^{-M}, \quad|\alpha| \leq r, \quad e \in E
$$

A2.

$$
\int_{\mathbb{R}^{d}} x^{\alpha} \psi^{e}(x) d x=0, \quad|\alpha|<r, \quad e \in E .
$$

Then the following two statements hold (see [FJW], [K3]):

Proposition 6.1. Let $s \in \mathbb{R}, 0<p<\infty, 0<q \leq \infty, \mathcal{J}:=d / \min \{1, p, q\}$, $r>\max \{\mathcal{J}-d-s, s\}$, and $M>\max \{\mathcal{J}, d+r\}$. Then, for every $f \in \dot{F}_{p q}^{s}$, there exist unique coefficients $c_{I e}(f),(I, e) \in D \times E$, such that

$$
f=\sum_{I \in D} \sum_{e \in E} c_{I e}(f) \psi_{I}^{e} \text { with } c_{I e}(f):=\left\langle f, \psi_{I}^{e}\right\rangle,
$$

where the convergence is considered in the sense of $\mathcal{S}^{\prime} / \mathcal{P}\left(\right.$ and in $\dot{F}_{p q}^{s}$ if $\left.q \neq \infty\right)$. Moreover,

$$
\|f\|_{\dot{F}_{p q}^{s}} \approx\left\|\left(\sum_{I, e}\left(|I|^{-s / d}\left|c_{I e}(f)\right| \lambda_{I}\right)^{q}\right)^{1 / q}\right\|_{L_{p}} .
$$

Proposition 6.2. Let $s \in \mathbb{R}, 0<p, q \leq \infty, \mathcal{J}:=d / \min \{1, p\}, r>\max \{\mathcal{J}-d-$ $s, s\}$ and $M>\max \{\mathcal{J}, d+r\}$. For every $f \in \dot{B}_{p q}^{s}$, there exist unique coefficients $c_{I e}(f),(I, e) \in D \times E$, such that

$$
f=\sum_{I \in D} \sum_{e \in E} c_{I e}(f) \psi_{I}^{e} \text { with } c_{I e}(f):=\left\langle f, \psi_{I}^{e}\right\rangle,
$$

where the convergence is considered in the sense of $\mathcal{S}^{\prime} / \mathcal{P}$ (and in $\dot{B}_{p q}^{s}$ if $\left.p, q \neq \infty\right)$. Moreover,

$$
\|f\|_{\dot{B}_{p q}^{s}} \approx\left(\sum_{e \in E} \sum_{m \in \mathbb{Z}}\left(\sum_{I \in D_{m}}\left(|I|^{-s / d+1 / p-1 / 2}\left|c_{I e}(f)\right|\right)^{p}\right)^{q / p}\right)^{1 / q} .
$$

- Two maximal inequalities. In the proof of Lemma 3.1 we use the FeffermanStein maximal inequality [FS]. We consider the maximal operator (see (3.5))

$$
M_{t}(f)(x):=\left(\sup _{Q \ni x}|Q|^{-1} \int_{Q}|f(y)|^{t} d y\right)^{1 / t} .
$$

Proposition 6.3. Let $0<p<\infty, 0<q \leq \infty$, and $0<t<\min \{p, q\}$. Then for any sequence of functions $\left(f_{j}\right)_{j \in \mathbb{Z}^{d}}$

$$
\left\|\left(\sum_{j \in \mathbb{Z}^{d}} M_{t}\left(f_{j}\right)^{q}\right)^{1 / q}\right\|_{L_{p}} \leq C\left\|\left(\sum_{j \in \mathbb{Z}^{d}}\left|f_{j}\right|^{q}\right)^{1 / q}\right\|_{L_{p}} .
$$

For the proof of Lemma 3.1, we also need the following lemma.

Lemma 6.1. Let $0<t \leq 1, M>d / t$, and $\mu \in \mathbb{Z}_{+}$. Then, for any sequence of complex numbers $\left(h_{I e}\right)_{(I, e) \in D_{\mu} \times E}$ and $x$ in the unit cube $I_{0}$, we have

$$
\sum_{\substack{I \in D_{\mu} \\ e \in E}}\left|h_{I e}\right|\left(1+\left|x_{I}\right|\right)^{-M} \leq C 2^{\mu d / t} M_{t}\left(\sum_{\substack{I \in D_{\mu} \\ e \in E}}\left|h_{I e}\right| \chi_{I}\right)(x) .
$$


Proof. We denote $Q_{j}:=\left[-2^{j}, 2^{j}\right)^{d}, \Omega_{0}:=Q_{0}$, and $\Omega_{j}:=Q_{j} \backslash Q_{j-1}, j=1,2, \ldots$ Let $\delta:=M / d-1 / t>0$. We have, for $x \in I_{0}$,

$$
\begin{aligned}
& \sum_{\substack{I \in D_{\mu} \\
e \in E}}\left|h_{I e}\right|\left(1+\left|x_{I}\right|\right)^{-M}=\sum_{j=0}^{\infty} \sum_{\substack{I \subset \Omega_{j} \\
e \in E}}\left|h_{I e}\right|\left(1+\left|x_{I}\right|\right)^{-M} \\
& \leq C \sum_{j=0}^{\infty} \sum_{\substack{I \subset \Omega_{j} \\
e \in E}}\left|h_{I e}\right| 2^{-j M}=C \sum_{j=0}^{\infty} 2^{-j d / t-j \delta d} \sum_{\substack{I \subset \Omega_{j} \\
e \in E}}\left|h_{I e}\right| \\
& \leq C \sup _{j \geq 0} 2^{-j d / t} \sum_{I \subset \Omega_{j}}\left|h_{I e}\right| \leq C\left(\sup _{j \geq 0} 2^{-j d} \sum_{\substack{I \subset Q_{j} \\
e \in E}}\left|h_{I e}\right|^{t}\right)^{1 / t} \\
& \leq C\left(\sup _{j \geq 0} 2^{-j d} 2^{\mu d} \int\left(\sum_{\substack{I \subset Q_{j} \\
e \in E}}\left|h_{I e}\right| \chi_{I}\right)^{t}\right)^{1 / t} \int^{1 / t} \\
& \left.\leq C 2^{\mu d / t}\left(\sup _{j \geq 0} \frac{1}{\left|Q_{j}\right|} \int_{\substack { Q_{j} \\
\begin{subarray}{c}{I \subset Q_{j} \\
e \in E{ Q _ { j } \\
\begin{subarray} { c } { I \subset Q _ { j } \\
e \in E } }\end{subarray}}\left|h_{I e}\right| \chi_{I}\right)^{t}\right)^{1 / t} \\
& \leq C 2^{\mu d / t} M_{t}\left(\sum_{\substack{I \in D_{\mu} \\
e \in E}}\left|h_{I e}\right| \chi_{I}\right)(x) .
\end{aligned}
$$

\section{ACKNOWLEDGMENTS}

The authors would like to thank Ronald DeVore for his suggestions for improvements and constant support.

\section{REFERENCES}

[BR] C. de Boor, A. Ron, Fourier analysis of the approximation power of principal shift invariant spaces Constr. Approx., 8 (1992), 427-462. MR 94c:41023

[Da] I. Daubechies Ten Lectures on Wavelets, SIAM, Philadelphia, 1992. MR 93e:42045

[De] R. DeVore, Nonlinear approximation Acta Numer. (1998), 51-150. MR 2001a:41034

[DJKP] D. Donoho, I. Johnstone, G. Kerkyacharian and D. Picard, Wavelet shrinkage asymptotia J. Royal Stat. Soc. Ser. B. 57 (1996), 301-369. MR 96g:62068

[DL] R. DeVore and G. G. Lorentz, Constructive Approximation, Springer, New York, 1993. MR 95f:41001

[Do] D. Donoho, Unconditional bases are optimal bases for data compression and for statistical estimation Appl. Comp. Harm. Anal., 1 (1993), 100-115. MR 94j:94011

[FJ1] M. Frazier, B. Jawerth, Decomposition of Besov Spaces Indiana Univ. Math. J., 34 (1985), 777-799. MR 87h:46083

[FJ2] M. Frazier, B. Jawerth, A discrete transform and decompositions of distribution, J. of Functional Analysis 93 (1990), 34-170. MR 92a:46042

[FJW] M. Frazier, B. Jawerth, and G. Weiss, Littlewood-Paley Theory and the Study of Function Spaces, CBMS 79 (1991), AMS. MR 92m:42021

[FS] C. Fefferman and E. Stein, Some maximal inequalities, Amer. J. Math. 93 (1971), 107115. MR 44:2026

[JM] R.-Q. Jia, C. Micchelli, Using the refinement equations for the construction of prewavelets II: powers of two, Curves and Surfaces, Academic Press, 1991. MR 93e:65024

[K1] G. Kyriazis, Wavelet coefficients measuring smoothness in $H_{p}\left(\mathbb{R}^{d}\right)$, Appl. Comp. Harm. Anal. 3 (1996), 100-119. MR 97h:42016 
[K2] G. Kyriazis, Approximation of Distribution Spaces by Means of Kernel Operators $J$. Four. Anal. Appl. 2 (1996), 261-286. MR 97d:46041

[K3] G. Kyriazis, Unconditional bases of function spaces, preprint.

[M] Y. Meyer, Ondelettes et Opérateurs I: Ondelettes, Hermann Éditeurs, 1990. MR 93i:42002

[Pee] J. Peetre, New thought on Besov spaces, Duke Univ. Math. Series. Durham, N.C., 1993. MR 57:1108

[Pek] A. Pekarskii, Chebyshev rational approximation in a disk, on a circle, and on a segment, Mat. Sb., 133 (175) (1987), 86-102, (English translation in Math. USSR Sbornik, 61 (1988), 87-102). MR 89e:30060

[Pet] P. Petrushev, Bases consisting of rational functions of uniformly bounded degrees or more general functions J. Funct. Anal., 174 (2000), 18-75. CMP 2000:13

[PP] P. Petrushev, V. Popov, Rational approximation of real functions, Cambridge University Press, Cambridge, 1987. MR 89i:41022

[T] H. Triebel, Theory of Function Spaces, Birkhäuser, 1983. MR 86j:46026

Department of Mathematics and Statistics, University of Cyprus, P. O. Box 20537, 1678 Nicosia, Cyprus

E-mail address: kyriazis@ucy.ac.cy

Department of Mathematics, University of South Carolina, Columbia, South CarOLINA 29208

E-mail address: pencho@math.sc.edu 